\title{
THEORY OF VOLTAGE DIVIDERS AND THEIR USE WITH CATHODE RAY OSCILLOGRAPHS
}

\author{
By Melville F. Peters, George F. Blackburn, and Paul T. Hannen
}

\section{ABSTRACT}

Four requirements are given for voltage dividers, and equations have been developed for the most general type of capacitance voltage divider. Special cases of the most general type are discussed. These equations show that if the capacitance of the voltage divider is small, the capacitance to ground can not be neglected if the deflection of the cathode beam is to be proportional to the applied voltage. A number of oscillograms of spark discharge using five types of circuits confirm the equations. It was found that resistors had to be placed in the voltage divider to damp out oscillations set up in the divider. Equations are given for resistance voltage dividers expressing the relation between the divided voltage and the applied voltage in terms of the resistance and self-capacitance of the resistors. The equations show that only under special conditions will the deflection of the cathode beam be proportional to the applied voltage. Oscillograms taken at frequencies of 20 to $1,000 \mathrm{kc}$ confirm the equations as clcsely as can be expected with the simple assumptions used in deriving them. Methods are given for the determination of the sensitivity of the cathode beam at the photographic plate, as well as the determination of the reduction ratio of the voltage divider.

I. Introduction

II. General requirements for a voltage divider

III. Analysis of voltage divider characteristics

1. Capacitance voltage divider

2. Resistance voltage divider

IV. Description of apparatus.

V. Experimental results with circuits 1 to 5

1. Method of balancing voltage divider to satisfy equation (5) - 97

2. Experimental verification of requirement 2 and equation $(6)_{-}-98$

3. Oscillograms showing the variation of voltage in magneto spark discharges ................................

VI. Experimental results with resistance voltage dividers.....

1. Alignment of the cathode tube 105

2. Sensitivity of the cathode beam 106

VIII. Calibration of the capacitance voltage divider

1. Calibration by application of known voltages.... 111

2. Calibration by use of the oscillator.

$\begin{aligned} \text { IX. Conclusion } & \\ \text { X. Acknowledgements. } & \end{aligned}$

\section{INTRODUCTION}

A fundamental requirement for an instrument designed to measure potential difference in an electric circuit is that the measuring instrument shall not introduce more than a negligible change in the character of the circuit under consideration. This sometimes imposes limiting conditions upon the instrument, especially in the case of measuring the voltage across a spark gap with a cathode ray oscillograph under conditions found in practice. 
In automotive ignition systems the capacitance and inductance are low. Measurements made on aircraft engines showed the capacitance of the leads to vary from 40 to $180 \mu \mu f$. On radio-shielded engines the capacitance is usually greater than this and has been found to be as high as $400 \mu \mu \mathrm{f}$. The proximity of the leads to the engine causes the inductance to change with frequency. At high frequencies the iron has very little importance and computations made for the shorter leads, disregarding the effect of iron, showed the inductance to be approximately $1 \mu \mathrm{h}$.

If the voltages are sufficiently low so that they may be applied directly to the deflection plates of the oscillograph, the latter increases the capacitance of the circuit by approximately $20 \mu \mu \mathrm{f}$. If a short lead is used from the magneto to the spark gap the combined capacitance of the circuit need not exceed $40 \mu \mu \mathrm{f}$, which enables the experimenter to set up a test circuit which is a sufficiently close approximation to the actual circuit as regards capacitance.

If the voltage is too high to be applied directly to the deflection plates a voltage divider must be used. In a study of magneto discharges it was found that a voltage divider was required, and one was designed which introduced a capacitance of $20 \mu \mu \mathrm{f}$. This voltage divider proved unsatisfactory and the present investigation was undertaken to study various possible arrangements and to determine which would be the most suitable for measuring the voltage characteristics of magneto spark discharges.

\section{GENERAL REQUIREMENTS FOR A VOLTAGE DIVIDER}

The oscillograph records the difference of potential between the two deflection plates at any instant. In order that the voltage recorded by the oscillograph shall be a true representation of the voltage to be measured it is necessary that the following conditions be satisfied.

1. Introduction of the voltage divider must not affect materially the character of the circuit.

2 . The potential difference across the deflection plates must at every instant be proportional to that across the divider, and the ratio must remain constant at all frequencies. This insures that the two potential differences will be in phase.

3 . The voltage between the deflection plates and ground must not be sufficient to cause a breakdown in the deflection tube or introduce spurious effects on the cathode beam.

4. There must be no natural oscillation in any part of the voltage divider which will produce spurious effects.

In the following discussion these requirements will be referred to by number.

\section{ANALYSIS OF VOLTAGE DIVIDER CHARACTERISTICS}

\section{CAPACITANCE VOLTAGE DIVIDER}

The most general type of capacitance voltage divider is shown diagrammatically in Figure 1. Referring to the figure let $A$ and $A^{\prime}$ be two points, which may be the electrodes of a sphere gap or spark plug, having potentials $V_{A}$ and $V_{A}^{\prime}$ with respect to ground. Let $V_{B}$ and $V_{B}^{\prime}$ be the potentials with respect to ground of the two deflection 
plates as indicated. Let $C_{1}$ and $C_{1}^{\prime}$ be the capacitances of the two series capacitors, and $C_{3}{ }^{\prime}$ the capacitance of the capacitor shunted across the deflection plates of the oscillograph. The capacitance of these plates is $C_{3}$. Let $C_{2}$ and $C_{2}^{\prime}$ be capacitances to ground, which are the effective capacitances to ground of the portion of the circuit metallically connected to $C_{2}$ and $C_{2}{ }^{\prime}$, respectively. By an inspection of Figure 1 equations (1) and (2) may be written

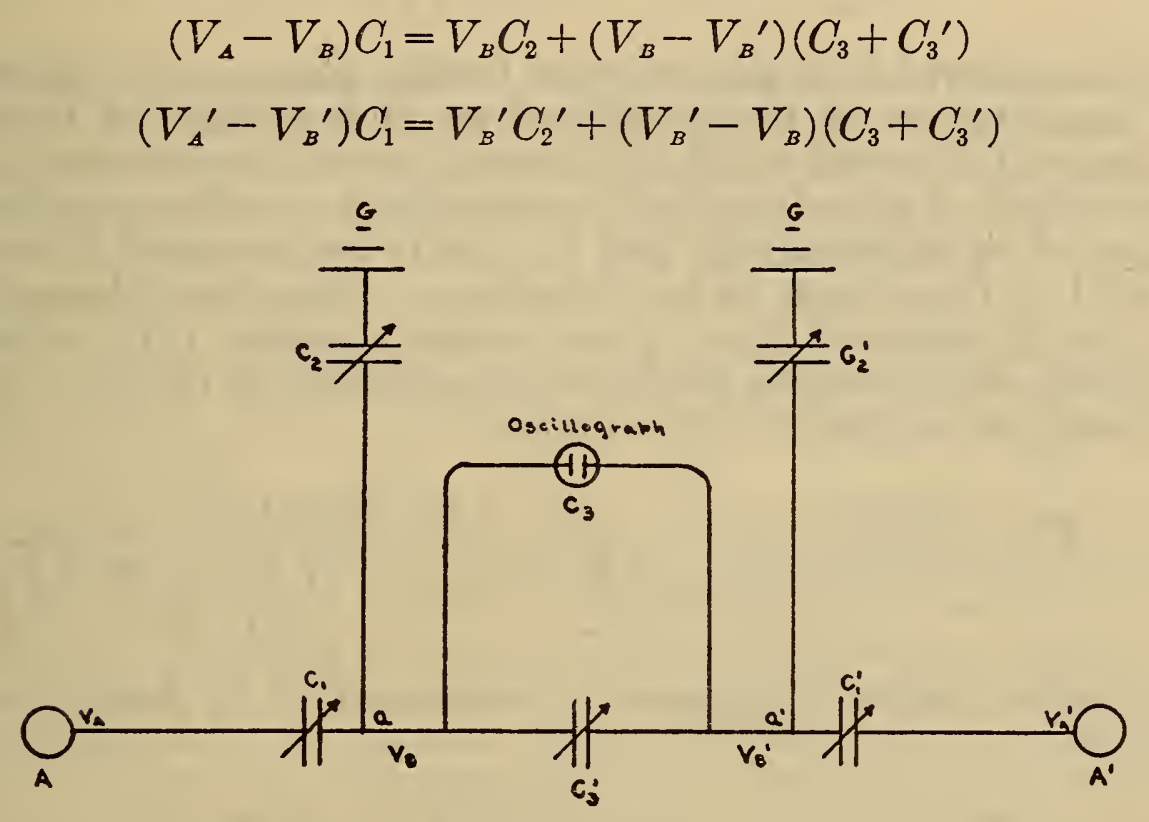

Figdre 1.-Capacitance voltage divider circuit

Subtracting (2) from (1) an expression is obtained for

$$
\begin{aligned}
V_{A}-V_{A}{ }^{\prime} & =V_{B}\left[1+\left(C_{3}+C_{3}^{\prime}\right)\left(\frac{1}{C_{1}}+\frac{1}{C_{1}^{\prime}}\right)+\frac{C_{2}}{C_{1}}\right] \\
-V_{B} & {\left[1+\left(C_{3}+C_{3}\right)\left(\frac{1}{C_{1}^{\prime}}+\frac{1}{C_{1}^{\prime}}\right)+\frac{C_{2}^{\prime}}{C_{1}^{\prime}}\right] }
\end{aligned}
$$

In a similar manner an expression may be obtained for $V_{B}-V_{B}^{\prime}$ which reduces to

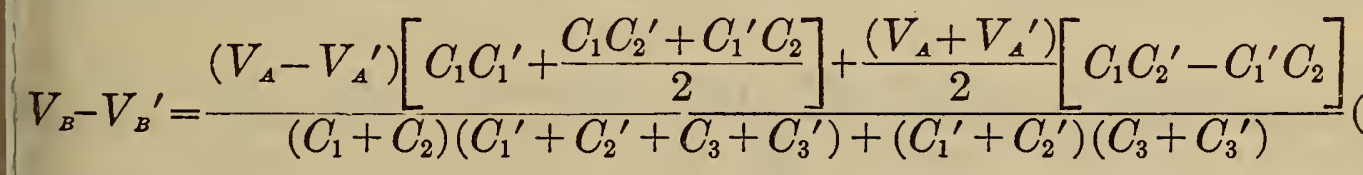

Equation (4) shows that the deflection which corresponds to $V_{B}-V_{B}^{\prime}$ will, in general, depend not only on the potential difference $V_{A}^{B}-V_{A}^{B}$, which is to be measured, but also on the average potential $\frac{V_{A}^{A}+V_{A}^{\prime}}{2}$ of the points $A$ and $A^{\prime}$ above ground. This latter effect can be liminated by adjusting the circuit to make

$$
\frac{C_{2}}{C_{1}}=\frac{C_{2}^{\prime}}{C_{1}^{\prime}}
$$


When this adjustment is made equations (3) and (4) may be written

where

$$
\frac{V_{B}-V_{B}^{\prime}}{V_{A}-V_{A}^{\prime}}=\frac{1}{K}
$$

$$
K=\left[1+\left(C_{3}+C_{3}^{\prime}\right)\left(\frac{1}{C_{1}}+\frac{1}{C_{1}^{\prime}}\right)+\frac{C_{2}}{C_{1}}\right]
$$

and will be referred to as the reduction factor. Equation (6) shows that if the capacitances of the capacitors are independent of frequency the voltage divider will satisfy the second general requirement.

The method of adjustment of the capacitors to satisfy equation (5) is suggested by equations (3) and (4), for if the potential $V_{A}$ is made equal to $V_{A}^{\prime}$, there must be no deflection of the cathode beam. This is true for all values of $V_{A}=V_{A}^{\prime}$ only when equation (5) is satisfied.

To obtain an expression for $V_{B}$ solve equation (1) for $V_{B}-V_{B}^{\prime}$ and substitute in equation (5). This gives

$$
V_{B}=\frac{V_{A} C_{1}}{C_{1}+C_{2}}-\frac{\left(V_{A}-V_{A}{ }^{\prime}\right)\left(C_{3}+C_{3}{ }^{\prime}\right)}{\left(C_{1}+C_{2}\right)\left[1+\left(C_{3}+C_{3}{ }^{\prime}\right)\left(\frac{1}{C_{1}}+\frac{1}{C_{1}{ }^{\prime}}\right)+\frac{C_{2}}{C_{1}}\right]}
$$

In a similar manner an expression is obtained for $V_{B}{ }^{\prime}$ from equations (2) and (5)

$$
V_{B}^{\prime}=\frac{V_{A}^{\prime} C_{1}^{\prime}}{C_{1}^{\prime}+C_{2}^{\prime}}+\frac{\left(V_{A}-V_{A}^{\prime}\right)\left(C_{3}+C_{3}^{\prime}\right)}{\left(C_{1}^{\prime}+C_{2}^{\prime}\right)\left[1+\left(C_{3}+C_{3}{ }^{\prime}\right)\left(\frac{1}{C_{1}}+\frac{1}{C_{1}^{\prime}}\right)+\frac{C_{2}^{\prime}}{C_{1}^{\prime}}\right]}
$$

Equations (7) and (8) give the potentials of the two defiection plates to ground in terms of the applied potential to ground and the electrical constants of the voltage divider. The maximum value which $V_{B}$ and $V_{B}^{\prime}$ may have to satisfy the third requirement depends upon the limitations imposed by the oscillograph. For this reason they must be estimated or determined experimentally, in which case,

it may be more desirable to solve equations (7) and (8) for $\frac{C_{2}}{C_{1}}$ and $\frac{C_{2}{ }^{\prime}}{C_{1}{ }^{\prime}}$ respectively, in terms of $\frac{\left(C_{3}+C_{3}{ }^{\prime}\right)}{C_{1}}, \frac{\left(C_{3}+C_{3}{ }^{\prime}\right)}{C_{1}{ }^{\prime}}$. This solution leads to the following relations:

$$
\begin{aligned}
& \frac{C_{2}}{C_{1}}=\frac{1}{2}\left[\frac{V_{A}}{V_{B}}-\left(C_{3}+C_{3}{ }^{\prime}\right)\left(\frac{1}{C_{1}}+\frac{1}{C_{1}^{\prime}}\right)-2\right] \\
& \begin{array}{c} 
\pm \frac{1}{2} \sqrt{\left(C_{3}+C_{3}{ }^{\prime}\right)^{2}\left(\frac{1}{C_{1}}+\frac{1}{C_{1}{ }^{\prime}}\right)^{2}+2\left(C_{3}+C_{3}{ }^{\prime}\right)\left(\begin{array}{c}
1 \\
\left.C_{1}+\frac{1}{C_{1}{ }^{\prime}}\right) \frac{V_{A}}{V_{B}}
\end{array}\right.} \\
-4 \frac{\left(C_{3}+C_{3}{ }^{\prime}\right)}{C_{1}} \frac{\left(V_{A}-V_{A}{ }^{\prime}\right)}{V_{B}}+\frac{V_{A}{ }^{2}}{V_{B}{ }^{2}}
\end{array}
\end{aligned}
$$




$$
\begin{gathered}
\frac{C_{2}^{\prime}}{C_{1}^{\prime}}=\frac{1}{2}\left[\frac{V_{A}^{\prime}}{V_{B}^{\prime}}-\left(C_{3}+C_{3}^{\prime}\right)\left(\frac{1}{C_{1}}+\frac{1}{C_{1}^{\prime}}\right)-2\right] \\
\sqrt{\frac{\left(C_{3}+C_{3}{ }^{\prime}\right)^{2}\left(\frac{1}{C_{1}}+\frac{1}{C_{1}^{\prime}}\right)^{2}+2\left(C_{3}+C_{3}^{\prime}\right)\left(\frac{1}{C_{1}}+\frac{1}{C_{1}^{\prime}}\right) \frac{V_{A}^{\prime}}{V_{B}^{\prime}}}{+4 \frac{\left(C_{3}+C_{3}^{\prime}\right)}{C_{1}^{\prime}} \frac{\left(V_{A}-V_{A}{ }^{\prime}\right)}{V_{B}}+\frac{V_{A}^{\prime 2}}{V_{B}^{\prime 2}}}}
\end{gathered}
$$

If the maximum potentials $V_{A}, V_{A}^{\prime}, V_{B}$ and $V_{B}^{\prime}$ are known approximately, the problem is to determine the five variable capacitors so as to obtain the desired deflection of the cathode beam and at the same time satisfy the four requirements. Methods of making calculations will now be given, and to simplify the presentation four cases will be considered governed by the maximum potentials of $V_{A}$ and $V_{A}^{\prime}$, and a fifth case pertaining to the elimination of oscillations in the voltage divider. The five corresponding circuits will be designated hereafter as circuits $1,2,3,4$, and 5 . They are shown diagrammatically in Figure 2.

An experimental study of these five circuits has been made at frequencies from 20 to $1,000 \mathrm{kc}$ per second. It was found that at frequencies above 1,000 kc care must be taken in the design and selection of the capacitors and that the drop in the leads from the source to the voltage divider, as well as in the leads in the voltage divider itself become important. It was also found that resonance occurred in the voltage divider when voltage at $10^{4} \mathrm{kc}$ frequency was applied across $A$ and $A^{\prime}$. This was first observed when sparking occurred across the plates of the capacitors $C_{1}$ and $C_{1}^{\prime}$, and later verified by connecting a milliammeter between $A$ and $C_{1}$. At resonance the milliammeter indicated a large current.

To eliminate repetition, certain characteristics of the circuits in the five cases are given.

1. To satisfy requirement 1 the maximum capacitance of $C_{1}$ was taken as $10 \mu \mu \mathrm{f}$.

2. The cathode beam was deflected $1 \mathrm{~cm}$ at the photographic plate for each 250 volts applied to the horizontal deflection plates. The maximum deflection which can be measured is approximately $5.6 \mathrm{~cm}$, so that $V_{B}-V_{B}{ }^{\prime}$ must not be greater than 1,400 volts.

3 . With a potential difference of 60,000 volts applied to the cathode tube the average potential gradient between cathode and anode is $\frac{60,000}{17.5}=3,500$ volts per centimeter. The distance between the two pairs of deflection plates is $11 \mathrm{~mm}$, and to insure against appreciable leakage from one pair to the other the average potential gradient between them was arbitrarily limited to half of that between cathode and anode. The potential difference between the pairs of deflection plates was accordingly limited to $\frac{3,500}{2} \times 1.1=1,925$ volts. Since the maximum potential of the oscillator plate to ground may be \pm 700 volts but was usually 500 volts, the greatest potential $V_{B}$ or $V_{B}^{\prime}$ should have is $1,925-500=1,425$ volts. This is also the potential of $V_{B}$ when $V_{B}^{\prime}=0$ and the deflection of the cathode beam at the photographic plate is $5.6 \mathrm{~cm}$. 
4. The capacitance $C_{3}$ in this treatment signifies the capacitance of the circuit metallically connected to one plate of the capacitor $C_{3}$ with respect to the circuit metallically connected to the other plate of $C_{3}$ with $C_{3}{ }^{\prime}$ omitted. The capacitance $C_{3}$ was estimated to be $25 \mu \mu \mathrm{f}$.
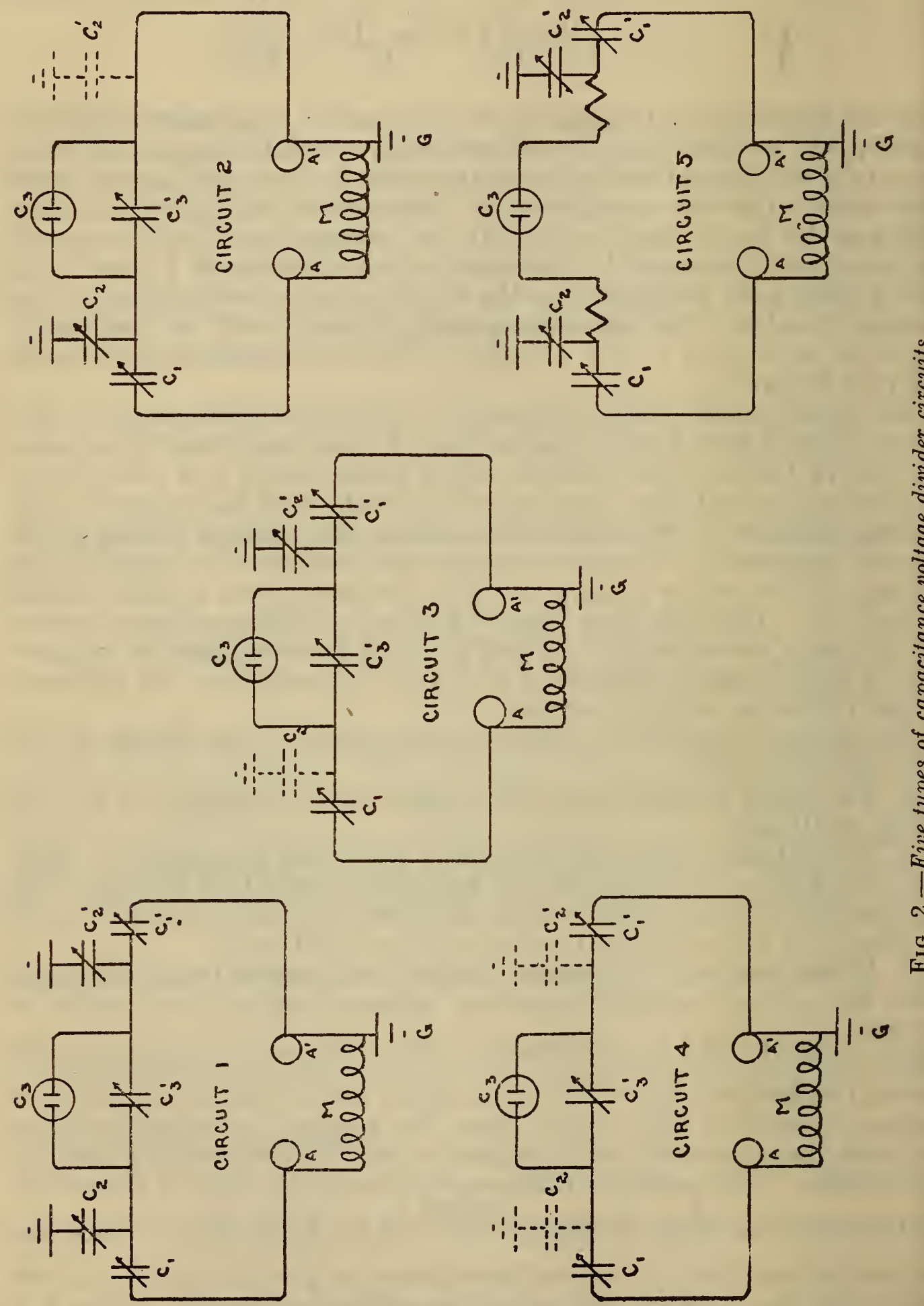

5. The capacitance $C_{2}$ signifies the capacitance to ground of the circuit consisting of the ungrounded plate of $C_{2}$ and the parts metallically connected to it, and $C_{2}^{\prime}$ has the corresponding meaning. It was estimated that when $C_{1}$ and $C_{1}^{\prime}$ had their maximum values of 10 
$\mu \mu \mathrm{f}$ each, the capacitances designated as $C_{2}$ and $C_{2}^{\prime}$ would not be less than $25 \mu \mu \mathrm{f}$ each, even though the capacitors $C_{2}$ and $C_{2}^{\prime}$ were removed.

Circuit 1. $V_{A}$ and $V_{A}^{\prime}$ large.- Let the maximum potential of $A$ be 10,000 volts and suppose that at the same instant $A^{\prime}$ has a maximum potential of 6,000 volts. To simplify the problem make the circuit symmetrical; that is, $C_{1}=C_{1}{ }^{\prime}, C_{2}=C_{2}^{\prime}$.

If the value $V_{A}-V_{A}^{\prime}=4,000$ volts be substituted in equation (6) it is found that in order to obtain a deflection of the cathode beam corresponding to 1,400 volts, $K$ must be equal to 2.86 .

It does not follow that it is permissible to adjust the circuit so that $K$ shall have this value, and it is found that with this value of $K$, it is not possible to satisfy the third requirement, concerning the maximum potentials of $V_{B}$ and $V_{B}^{\prime}$. It is therefore necessary to use a larger value of $K$; that is, to work with less than the full deflection of the cathode beam.

To find the smallest value of $K$ which makes it possible to satisfy the first three requirements it is necessary to use equation (9). Substituting in equation (9) the values

$$
\frac{V_{A}}{V_{B}}=\frac{10,000}{1,400}=7.14, \frac{V_{A}-V_{A}^{\prime}}{V_{B}}=\frac{10,000-6,000}{1,400}=2.86, C_{1}=C_{1}^{\prime}
$$

and plotting $\frac{C_{2}}{C_{1}}$ versus $\frac{C_{3}+C_{3}{ }^{\prime}}{C_{1}}$ the curve shown at $(a)$ in Figure 3 was obtained. From this curve may be obtained the value which $\frac{C_{2}}{C_{1}}$ must have when $\frac{C_{3}+C_{3}^{\prime}}{C_{1}}$ is selected, or vice versa, in order that $V_{B}$ shall be equal to 1,400 volts when $V_{A}$ and $V_{A}^{\prime}$ have their maximum values. If $K$ is computed from curve $(a)$ and the value plotted versus $\frac{C_{3}+C_{3}^{\prime}}{C_{1}}$ the curve shown at $(b)$ is obtained. This curve shows that the smallest value of $K$ is obtained when $\frac{C_{3}+C_{3}{ }^{\prime}}{C_{1}}=0$, and the value of $K$ increases as $\frac{C_{3}+C_{3}{ }^{\prime}}{C_{1}}$ increases. To make $\frac{C_{3}+C_{3}{ }^{\prime}}{C_{1}}$ as small as possible $C_{3}{ }^{\prime}$ is made zero, and $C_{1}$ is given its largest value of $10 \mu \mu \mathrm{f}$. $C_{3}$ is constant and equal to $25 \mu \mu$ so that the smallest value of $\frac{C_{3}+C_{3}{ }^{\prime}}{C_{1}}$ is $\frac{0+25}{10}=2.5$. The corresponding value of $\frac{C_{2}}{C_{1}}$, curve $(a)$, is 5.52, rence $C_{2}=55.2 \mu \mu \mathrm{f}$ and $K=11.52$. Substituting this value of $K$ in equation (6), $V_{B}-V_{B}^{\prime}=347$ volts, which is the maximum possible potential difference across the deflection plates when all the requirenents are complied with.

Since this corresponds to a deflection of less than $2 \mathrm{~cm}$ at the photographic plate it is worth while to consider whether this deflection sould be appreciably increased by removing the restrictions on the values of $V_{B}$ and $V_{B}^{\prime}$. Plotting $V_{B}$ and $V_{B}^{\prime}$ as given by equations (7) and (8) with $C_{2}$ as the independent variable the two curves shown at a) and $(b)$ in Figure 4 were obtained. Determining $V_{B}-V_{B}{ }^{\prime}$ from 3urves $(a)$ and $(b)$ for a number of values of $C_{2}$, the curve shown at c) was plotted. This curve shows that with the value $C_{1}=C_{1}^{\prime}=$ $10 \mu \mu \mathrm{f}, C_{3}+C_{3}{ }^{\prime}=25 \mu \mu \mathrm{f}$, and the smallest value of $C_{2}=C_{2}{ }^{\prime}=25 \mu \mu \mathrm{f}$, 
the greatest deflection which can be obtained at the photographic plate corresponds to 450 volts at the deflection plates. Curve $(a)$ shows however that $V_{B}$ would be 2,500 volts, and since, as has been mentioned previously, the other pair of deflection plates may have a potential to ground of opposite sign and amounting to 500 volts, the

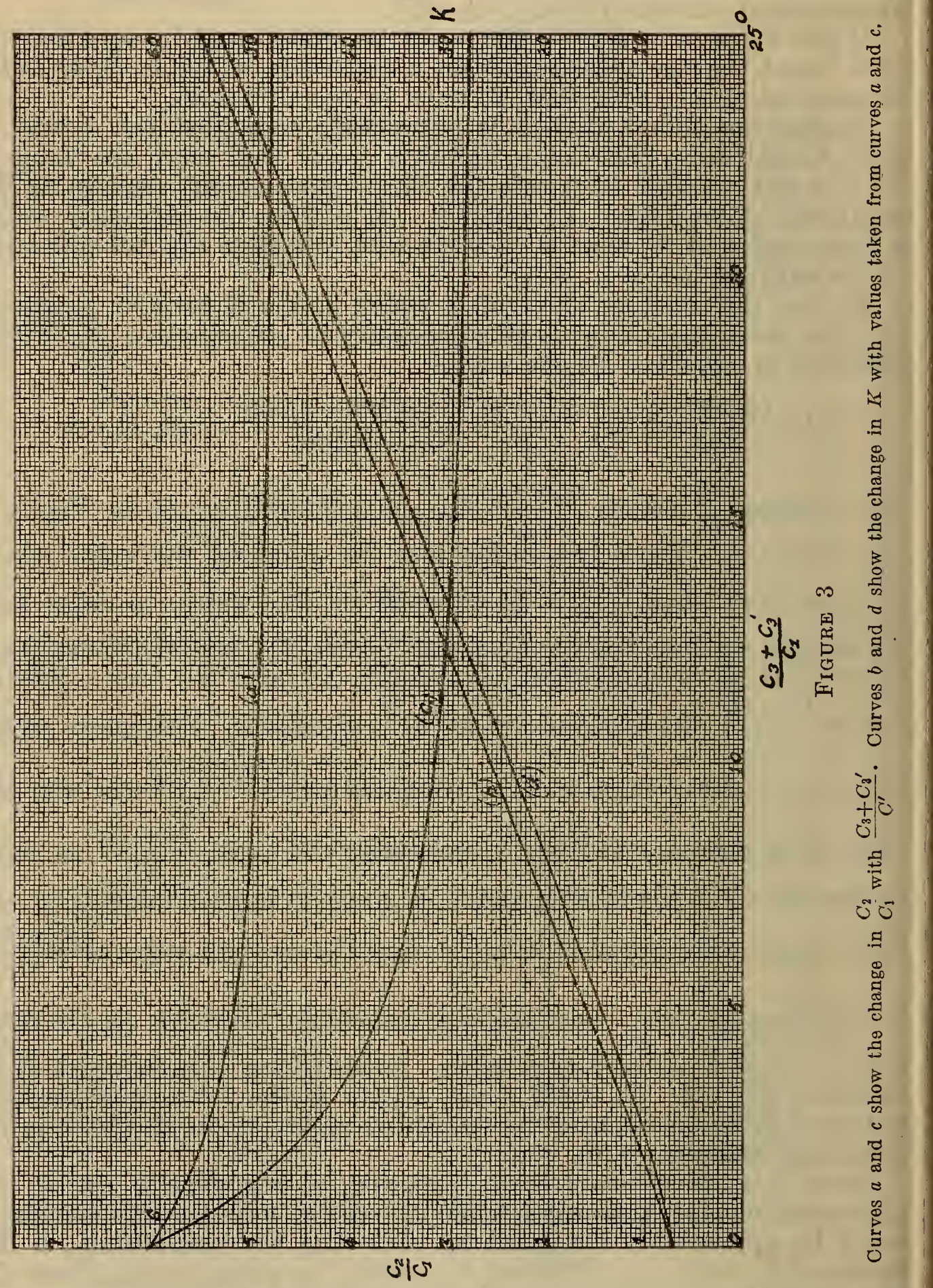

potential difference between the pairs of plates might be sufficient to cause a discharge to take place between them.

The manner in which the capacitors $C_{1} ; C_{1}{ }^{\prime}$, and $C_{3}{ }^{\prime}$, may be varied to increase the deflection are obvious and need not be further considered. 
If $V_{A}^{\prime}$ becomes negative to $V_{A}$ the greatest voltage which may be measured is $K\left(V_{B}-V_{B}^{\prime}\right)=11.5 \times 1,400=16,100$. Thus, if the absolute value of $V_{A}$ is not greater than 10,000 volts, $V_{A}{ }^{\prime}$ may have any absolute value not exceeding 6,100 volts. Since the arrangement is symmetrical the absolute potential $V_{A}^{\prime}$ may be 10,000 volts if the absolute potential of $V_{A}$ does not exceed 6,100 volts.

Circuit 2. $V_{A}$ large, $V_{A}{ }^{\prime}=0$. - If computations for circuit 1 are made for $V_{A}=10,000$ volts and $V_{A}^{\prime}=0$, instead of 6,000 volts, the maximum

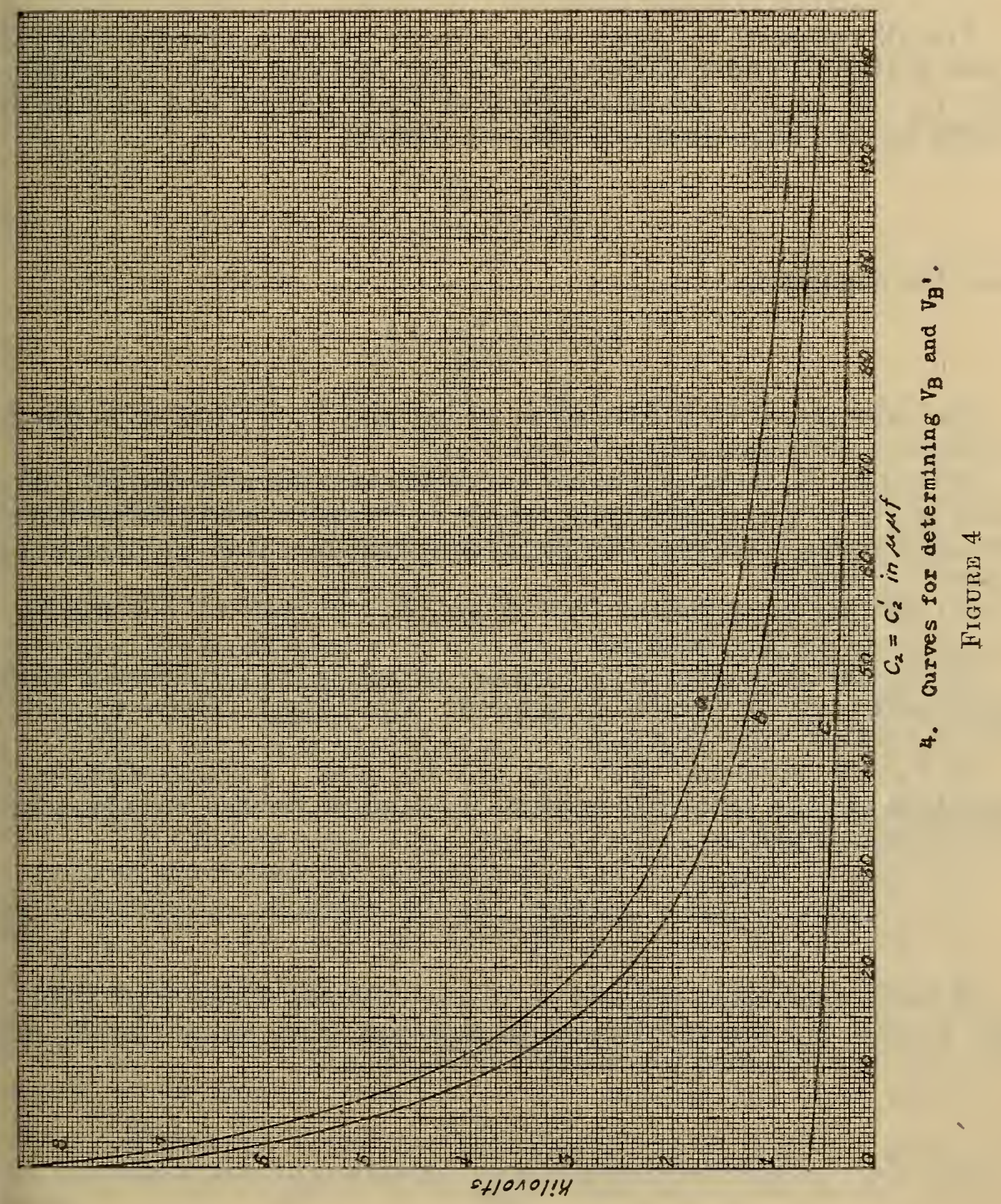

potential difference across the deflection plates is 870 . With the data yiven $C_{2}$ may be determined from equation (9). Curves $(c)$ and $(d)$ are shown in Figure 3 with $V_{A}^{\prime}=0$, so that a comparison may be made with curves $(a)$ and $(b)$ which were calculated with $V_{A}=6,000$ volts. If $C_{2}, C_{2}^{\prime}$ and $C_{3}+C_{3}^{\prime}$ are given their smallest capacitance, $25 \mu \mathrm{f}$ each, and $C_{1}, C_{1}^{\prime}$, their largest capacitance, $10 \mu \mu \mathrm{f}$ each, the rreatest potential difference $V_{B}-V_{B}{ }^{\prime}$ is $\frac{10,000}{10.25}=975$ volts. In order to 
get a greater deflection it is customary to make $C_{1}{ }^{\prime}=\infty$. Equation (3) becomes

$$
V_{A}-V_{A}^{\prime}=V_{B}\left[1+\left(C_{3}+C_{3}^{\prime}\right)\left(\frac{1}{C_{1}}\right)+\frac{C_{2}}{C_{1}}\right]-V_{B}^{\prime}\left[1+\left(C_{3}+C_{3}{ }^{\prime}\right)\left(\frac{1}{C_{1}}\right)\right]
$$

and since $V_{B}{ }^{\prime}=V_{A}^{\prime}=0$

$$
\frac{V_{B}}{V_{A}}=\frac{C_{1}}{C_{1}+C_{2}+C_{3}+C_{3}^{\prime}}
$$

For all values of $V_{B}{ }^{\prime}$ other than zero the relation expressed by (12) does not hold, and requirement 2 is not satisfied. The capacitance $C_{2}+C_{3}+C_{3}^{\prime}$ is now the capacitance of $V_{B}$ to ground and may be denoted by $C_{G}$. Equation (12) may be written

$$
\frac{V_{B}}{V_{A}}=\frac{C_{1}}{C_{1}+C_{G}}
$$

and the capacitance

$$
C_{G}=C_{1}\left(\frac{V_{A}}{V_{B}}-1\right)
$$

For example, if $C_{1}$ is limited to $10 \mu \mu$ and $V_{B}=1,400$ volts for the maximum deflection, then $C_{G}=61.4 \mu \mu \mathrm{f}$.

Circuit 3. $V_{A}$ large, $V_{A}^{\prime}$ small.-Let the maximum potential difference of $V_{A}-V_{A}^{\prime}=10,000$ volts, where $V_{A}^{\prime}$ may take any value from 0 to 1,000 volts. Consider two possible arrangements of circuit 2 designated $(a)$ and $(b)$ :

(a) In this arrangement $C_{1}=10 \mu \mu \mathrm{f}, C_{3}=25 \mu \mu \mathrm{f}, C_{3}{ }^{\prime}=0$ and $C_{2}=$ $C_{G}-C_{3}=61.4-25=36.4 \mu \mu \mathrm{f}$.

(b) In this arrangement $C_{2}=25 \mu \mu \mathrm{f}$, which is the capacitance of the leads and capacitor plates to ground. $C_{1}=10 \mu \mu \mathrm{f}$. Since $C_{G}=61.4$, $C_{3}+C_{3}{ }^{\prime}=61.4-25=36.4 \mu \mu$.

If $V_{A}^{\prime} \pm 0$, equation (12) can not be used. Putting $C_{1}{ }^{\prime}=\infty$ in equation (4) the following relation is obtained for the voltage across the deflection plates.

$$
V_{B}-V_{B}^{\prime}=\frac{V_{A} C_{1}-V_{A}^{\prime}\left(C_{1}+C_{2}\right)}{C_{1}+C_{2}+C_{3}+C_{3}^{\prime}}
$$

\begin{tabular}{|c|c|c|c|c|c|}
\hline \multirow{2}{*}{$V_{A}$} & \multirow{2}{*}{$V_{A^{\prime}}=V_{B^{\prime}}$} & \multicolumn{2}{|c|}{ (a) } & \multicolumn{2}{|c|}{ (b) } \\
\hline & & $V_{B}$ & $V_{B}-V_{B^{\prime}}$ & $V_{B}$ & $V_{B}-V_{B}^{\prime}$ \\
\hline 10,000 & 0 & 1,400 & 1,400 & 1,400 & 1,400 \\
\hline 10,200 & 200 & 1,490 & 1,290 & 1,530 & 1,330 \\
\hline 10,400 & & 1,590 & 1,190 & $\begin{array}{l}1,660 \\
1,700\end{array}$ & 1,260 \\
\hline $\begin{array}{l}10,600 \\
10.800\end{array}$ & $\begin{array}{l}600 \\
800\end{array}$ & $\begin{array}{l}1,690 \\
1,790\end{array}$ & 1,090 & $\begin{array}{l}1,790 \\
1,920\end{array}$ & $\begin{array}{l}1,190 \\
1,120\end{array}$ \\
\hline 11,000 & 1,000 & 1,800 & 890 & 2,050 & 1,050 \\
\hline
\end{tabular}

If the potentials to ground shown in columns 1 and 2 of Table 1 for $V_{A}$ and $V_{A}^{\prime}$ are substituted in equation (15), values for $V_{B}$ and $V_{B}-V_{B}^{\prime}$ are obtained for the two arrangements.

TABLE 1 
These computations show that unless $V_{A}^{\prime}$ remains at ground potential the deflection is not proportional to the applied potential difference.

When the magneto is used as a source of voltage $V_{A}^{\prime}$ is usually small compared to $V_{\Lambda}$ until the spark gap breaks down. If peakvoltage measurements are to be made the capacitance $C_{1}{ }^{\prime}$ may be made large in which case $C_{2}^{\prime}$ is also increased so that $\frac{C_{2}}{C_{1}}=\frac{C_{2}^{\prime}}{C_{1}^{\prime}}$. Table 2 gives the values for $V_{B}$ and $V_{B}-V_{B}{ }^{\prime}$ using circuit 1 with the following capacitances.

(c) $C_{1}=C_{1}{ }^{\prime}=10 \mu \mu \mathrm{f}, C_{2}=C_{2}{ }^{\prime}=44: 3 \mu \mu \mathrm{f}, C_{3}=25 \mu \mu \mathrm{f}$ and $C_{3}{ }^{\prime}=0$, which are the same values given in circuit 2 .

(d) $C_{1}=10 \mu \mu \mathrm{f}, C_{1}{ }^{\prime}=60 \mu \mu \mathrm{f}, C_{2}=38.5 \mu \mu \mathrm{f}, C_{2}{ }^{\prime}=231 \mu \mu \mathrm{f}, C_{3}=25$ $\mu \mu \mathrm{f}$, and $C_{3}{ }^{\prime}=0$.

TABLE 2

\begin{tabular}{|c|c|c|c|c|c|}
\hline \multirow{2}{*}{$V_{\Delta}$} & \multirow{2}{*}{$V_{\Delta^{\prime}}$} & \multicolumn{2}{|c|}{ (c) } & \multicolumn{2}{|c|}{ (d) } \\
\hline & & $V_{B}$ & $V_{B}-V_{B^{\prime}}$ & $V_{B}$ & $V_{B}-V_{B^{\prime}}$ \\
\hline $\begin{array}{l}10,000 \\
11,000\end{array}$ & $\begin{array}{r}0 \\
1,000\end{array}$ & $\begin{array}{l}1,400 \\
1,580\end{array}$ & $\begin{array}{l}960 \\
960\end{array}$ & $\begin{array}{l}1,400 \\
1,600\end{array}$ & $\begin{array}{l}1,290 \\
1,290\end{array}$ \\
\hline
\end{tabular}

In both $(c)$ and $(d)$ the deflection is proportional to the applied potential difference. At low frequencies, arrangement $(d)$ is superior to arrangement $(c)$. At high frequencies the large capacitance of $C_{1}{ }^{\prime}$, $C_{2}^{\prime}$ to ground which is $\frac{C_{1}^{\prime} \times C_{2}^{\prime}}{C_{1}+C_{2}^{\prime}}$ will allow large currents to flow and the drop in the leads from $A^{\prime}$ to $C_{1}{ }^{\prime}$ and $C_{1}{ }^{\prime}$ to $C_{2}{ }^{\prime}$ will affect the voltage across the deflection plates. At frequencies not greater than $10^{3} \mathrm{kc}$ this should not be serious when $V_{A}^{\prime}$ is small. This circuit can be used to advantage when it is uncertain as to whether or not $V_{A}^{\prime}$ remains at zero potential.

Circuit 4. $\dot{V}_{A}$ and $V_{A}^{\prime}$ both small.-When $V_{A}$ and $V_{A}^{\prime}$ are both small, but $V_{A}-V_{A}^{\prime}$ exceeds 1,400 volts, the capacitances $C_{2}$ and $C_{2}^{\prime}$ are made as small as practicable by omitting the two capacitors $C_{2}$ and $C_{2}{ }^{\prime}$.

Substituting in equation (8) the limiting values previously mentioned $C_{1}=C_{1}{ }^{\prime}=10 \mu \mu \mathrm{f}, C_{2}=C_{2}{ }^{\prime}=25 \mu \mu \mathrm{f}$, and $C_{3}+C_{3}{ }^{\prime}=25 \mu \mu \mathrm{f}$, $\frac{V_{B}-V_{B}^{\prime}}{V_{A}-V_{A}^{\prime}}=1 / 8$, which is the largest value $1 / K$ may have with this arrangement under the conditions specified. If $C_{1}$ and $C_{1}^{\prime}$ are increased, $\frac{C_{2}}{C_{1}}, \frac{C_{2}^{\prime}}{C_{1}^{\prime}}$ and $\left(C_{3}+C_{3}^{\prime}\right)\left(\frac{1}{C_{1}}+\frac{1}{C_{1}^{\prime}}\right)$ become less, and $1 / K$ increases. Rogowski, Wolff, and Klemperer ${ }^{1}$ have described this circuit for which the capacitances of $C_{1},\left(\mathrm{C}_{3}+C_{3}{ }^{\prime}\right)$, and $C_{1}^{\prime}$ are much greater.

Circuit 5. Aperiodic voltage divider circuit.-If the voltage divider is used to measure spark discharges, oscillations may be expected in any circuit where $\frac{R^{2}}{4 L^{2}}\left\langle\frac{1}{L C}\right.$. Such oscillations were present and the cir-

'Rogowski, Wolff, and Klemperer: “Die Spannungsteilen Kathodenoszillographen.” Archiv für Elekrotechnik, vol. 23 , p. $579,1929-30$. 
cuit was made aperiodic by inserting resistors at $(a)$ and $\left(a^{\prime}\right)$, (fig. 1 or 2) so as to make $\frac{R^{2}}{4 L^{2}}>\frac{1}{L C}$, where $L$ is the inductance of the lead $C_{1} C_{3}$, and $C$ the smaller of the two capacitances $C_{1}$ and $C_{3}$. In this manner requirement 4 is satisfied.

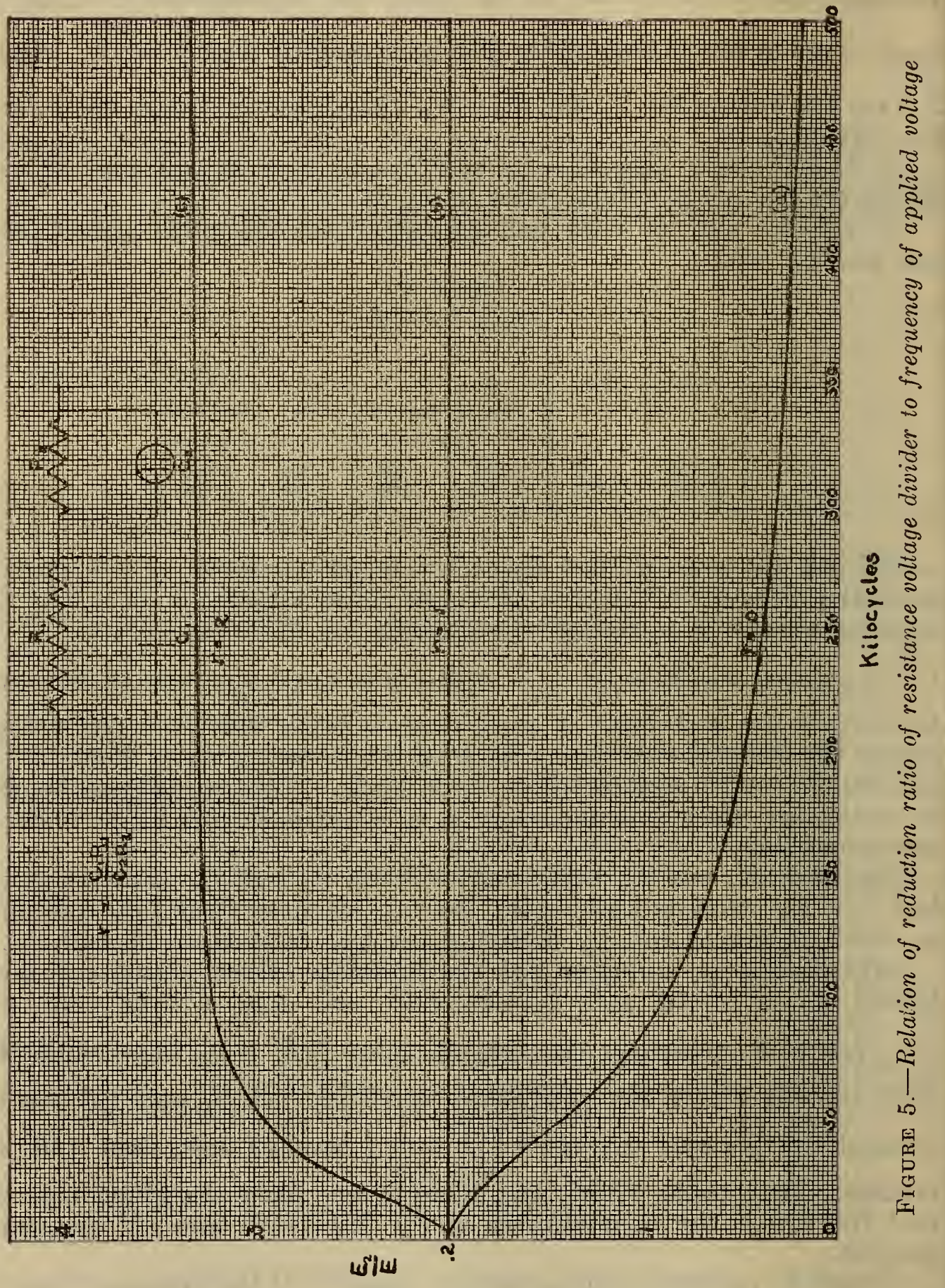

\section{RESISTANCE VOLTAGE DIVIDERS}

The resistance voltage divider arrangement is shown in Figure 5. At low frequencies the impedance of the deflection plates is so high compared with that of the portion $R_{2}$ of the voltage divider that only a small part of the current is shunted to the plates, so that the ratio of the voltage to be measured to that across the deflection plates is 
independent of frequency. The difference in phase between the two is also small. Computations which follow show that in order to keep the reduction ratio constant as the frequency is increased, resistance of the divider must be decreased, and at frequencies found in spark discharges a suitable resistance would be so low as to prevent a spark passing across the gap. If the resistance has a small capacitance and inductance the relation becomes more complicated. When grid leaks are used for resistance the inductance is small, whereas the capacitance across the grid leaks when supported in holders becomes important at high frequencies. Neglecting the inductance of the resistors and their capacitance to ground, an equation showing the relation between the voltage applied to the divider and the voltage across the deflection plates may be determined in terms of the resistance and capacitance of each portion of the voltage divider.

Let $R_{1}$ and $R_{2}$ designate the resistances of the resistors shown in the diagram of Figure 5, and let $C_{1}$ represent the capacitance of the resistor $R_{1}$, and $C_{2}$ the combined capacitance of the resistor $R_{2}$ and the oscillograph deflection plates. The impedance of each branch may be written

$$
Z_{1}=\frac{R_{1}}{1+j \omega C_{1} R_{1}} \text { and } Z_{2}=\frac{R_{2}}{1+j \omega C_{2} R_{2}}
$$

where $\omega$ is $2 \pi$ times the frequency $\eta$ and $j=\sqrt{-1}$. The total impedance for the circuit is

$$
Z=\frac{R_{1}}{1+j \omega C_{1} R_{1}}+\frac{R_{2}}{1+j \omega C_{2} R_{2}}
$$

and since $E=I Z$, where $E$ is the voltage across the divider and $I$ the current, the relation becomes

which reduces to

$$
\frac{E}{E_{2}}=\frac{\frac{R_{1}}{1+j \omega C_{1} R_{1}}+\frac{R_{2}}{1+j \omega C_{2} R_{2}}}{\frac{R_{2}}{1+j \omega C_{2} R_{2}}}
$$

$$
\frac{E_{2}}{E}=\frac{1}{\sqrt{\left[\frac{R_{1}\left(1+\omega^{2} C_{1} C_{2} R_{1} R_{2}\right)}{R_{2}\left(1+\omega C_{1} R_{1}^{2}\right)}+1\right]^{2}+\left[\frac{\omega R_{1}\left(C_{2} R_{2}-C_{1} R_{1}\right)}{R_{2}\left(1+\omega C_{1} R_{1}^{2}\right)}\right]^{2}}}
$$

which expresses the relation between the voltage across the deflection plates and that applied to the voltage divider.

If the resistance has no capacitance $C_{1}=0$ and equation (16) reduces to

$$
\frac{E_{2}}{E}=\frac{1}{\sqrt{\left[\frac{R_{1}}{R_{2}}+1\right]^{2}+\left[\omega C_{2} R_{1}\right]^{2}}}
$$

If $C_{1} R_{1}=C_{2} R_{2}$, equation (16) becomes independent of frequency since it reduces to

$$
\frac{E_{2}}{E}=\frac{1}{\frac{R_{1}}{R_{2}}+1}=\frac{R_{2}}{R_{1}+R_{2}}
$$


These equations show that whether $\frac{E_{2}}{E}$ will increase, decrease, or not change with frequency depends upon the values of $R_{1}, R_{2}, C_{1}$ and $C_{2}$. Putting $r=\frac{C_{1} R_{1}}{C_{2} R_{2}}, R_{1}=8 \times 10^{5}$ ohms, $R_{2}=2 \times 10^{5}$ ohms, $C_{2}=20 \mu \mu f$, and assigning to $C_{1}$ the values of 0,5 , and $10 \mu \mu f$ so that $r$ takes the corresponding values of 0,1 , and 2 the curves shown in Figure 5 were obtained. In these curves $(a)$ was plotted from equation (17), showing that the ratio decreases very rapidly with increase in frequency. As $\omega$ increases without limit $\frac{E_{2}}{E}$ approaches 0 . Curve $(b)$ is a plot of equation (18) which shows that the voltage divider is independent of frequency when $r=1$. Curve $(c)$ is a plot of equation (16) and shows that the ratio $\frac{E_{2}}{E}$ increases with an increase in $\omega$. As $\omega$ approaches $\infty, \frac{E_{2}}{E}$ approaches the limiting value $\frac{C_{1}}{C_{1}+C_{2}}$.

If the capacitance $C_{1}$ is assumed to be so small it may be neglected, computations from equation (17) show that in order that $\frac{E_{2}}{E}=0.2$ shall not diminish by more than 5 per cent (or not be less than 0.19), $\omega^{2} C^{2} R_{1}{ }^{2}$ must not be greater than 2.56. Since $C_{2}=20 \times 10^{-12} \mathrm{f}$ (which includes the leads as well as the defiection plates), $\omega^{2} R_{1}{ }^{2}=2.56 \times \frac{10^{22}}{4}$ $=6.4 \times 10^{21}$. If $\omega=10^{7}$ or $\eta=\frac{10^{7}}{2 \pi}, R_{1}{ }^{2}=\frac{6.4 \times 10^{21}}{10^{14}}=6.4 \times 10^{7}$. Therefore, $R_{1}=8,000$ ohms, $R_{2}=2,000$ ohms and $R_{1}+R_{2}=10,000$ ohms, which is the maximum resistance which may be used at a frequency of $\frac{10^{7}}{2 \pi}$ or approximately $1,600 \mathrm{kc}$. Such a resistance across the gap would prevent sparking. Silsbee ${ }^{2}$ has shown that a resistance of $5 \times 10^{4} \mathrm{ohms}$ is so low as to seriously affect the character of the discharge and might actually prevent the occurrence of a spark. For high-compression engines such as are in use to-day the resistance must be much greater than this. Such low resistances are objectionable in this work because they affect the character of the spark. At times it may happen that fouling of plugs will reduce the resistance across a spark plug to $5 \times 10^{4} \mathrm{ohms}$, but this is a special condition, and while an interesting one, should not be a limiting condition of the problem.

The remainder of this report is devoted to an experimental study of the theory developed in this and the preceding sections, together with a discussion of oscillograph technique and the calibration of the voltage divider.

\section{DESCRIPTION OF APPARATUS}

The cathode ray oscillograph was of the high-voltage cold-cathode type. The cathode was operated at approximately 60,000 volts, the supply being the secondary of a high tension transformer. The primary was connected through rotary switches to a 60 -cycle 110 -volt

${ }^{2}$ Silsbee, N. A. C. A. Report No. 241, Electrical Characteristics of Spark Generators for Automotive Ignition, p. 27. 
supply, the switches being operated by a synchronous motor driven from the same source. The switches were constructed so that the 60,000 volts was applied to the cathode on the negative half cycle, either once in 20 cycles or once in 100 cycles. For the present investigation the slower rate of excitation was used.

The cathode tube ( $E D$, fig. 6) was modified so that the cathode could be removed and polished. This was made possible by fusing the tungsten rod supporting the cathode to the movable portion of the ground joint as shown in the figure. The diaphragm $D$ was mounted in the glass tube so that both the cathode and diaphragm moved together.

The deflection tube has two pairs of deflection plates, one pair horizontal and the other pair vertical. They are shown at $A$ and $B$ in Figure $6 . \quad C$ is a coil which produces a magnetic field deflecting the cathode beam in a horizontal direction. If observations are to be made at low frequencies the voltage is applied to plates $A$, producing a vertical deflection. If, at the same time, the cathode beam is swept across the field by the magnetic sweep $C$, a record is obtained which

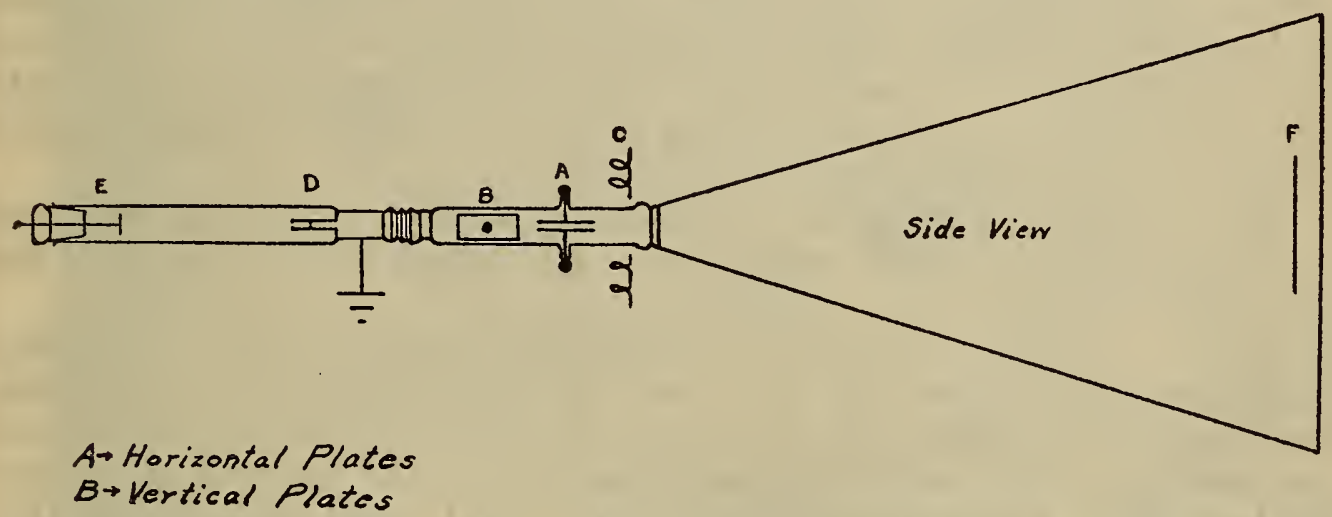

FigURE 6.-Diagram of cathode ray oscillograph

shows the variation with time of the voltage across the deflection plates. An oscillator with a range of 20 to $1,000 \mathrm{kc}$ was supplied with the oscillograph. If this oscillator is connected to plates $A$ and the sweep actuated, a sine wave is obtained. For high frequencies the voltage to be measured is connected to plates $B$, and superimposes upon the sine wave a horizontal deflection proportional to the voltage across the plates. The sine wave then gives the time coordinate.

With 60,000 volts applied to the cathode the deflection of the beam at the photographic plate is approximately $1 \mathrm{~cm}$ for each 250 volts applied to the horizontal deflection plates. The maximum cathode beam deflection which can be photographed is $5.6 \mathrm{~cm}$, thus limiting the useful voltage across the deflection plates to about 1,400.

The longest time required to sweep the beam across the photographic plate is 1,200 microseconds, and when necessary a faster sweep is used. If the spark discharge is to be photographed in the center of the film the magneto has to be timed accurately. This is accomplished by driving the magneto with the synchronous motor through a set of gears. Adjustment of the gears allows for approximate timing, and the final adjustment is made with the timing device on the magneto. The magneto is radio shielded and was designed for use with 12-cylinder aircraft engines. 
Figure 7 is a photograph showing the arrangement of the five capacitors $C_{1}, C_{1}^{\prime}, C_{2}, C_{2}^{\prime}$, and $C_{3}^{\prime} . \quad C_{1}$ and $C_{1}^{\prime}$ are two high-voltage radio transmitting capacitors. The range of capacitance is from 10 to $80 \mu \mu f . C_{2}$ and $C_{2}^{\prime}$ are two high-voltage radio transmitting capacitors having a range of capacitance from 40 to $300 \mu \mu \mathrm{f}$. Since the maximum voltage across $C_{2}$ and $C_{2}^{\prime}$ is 1,400 , it is not necessary to use high-voltage capacitors. It is necessary to keep the leakage of the portion of the circuit called $C_{2}$ and $C_{2}^{\prime}$ in Section III small, and this requires that the capacitors in the circuit have a high insulation resistance. Since the high-voltage capacitors had a much higher insulation resistance than the low-voltage capacitors which were available, the former were used. $C_{3}{ }^{\prime}$ was made by removing alternate plates from a low-voltage capacitor and supporting as shown in the photograph. The range of capacitance was 10 to $80 \mu \mu f$.

To further reduce leakage the bakelite binding posts were removed and the lead wires brought to the oscillograph through 1-inch holes in the case. This at first seemed an unnecessary precaution, but it was found that the resistance of the binding posts to ground was much less than that of the remainder of the insulated circuit. In magneto discharges a, small potential difference remains across the gap between discharges, and although the discharges alternate in sign, this additional leakage through the binding posts was sufficient to noticeably affect the results.

The five circuits may be obtained by simply removing or short circuiting the necessary capacitors. In circuit 5 the resistors are inserted at $(a)$ and $\left(a^{\prime}\right)$.

The leads shown at $A D$ and $A^{\prime} D^{\prime}$ should be fixed when the voltage divider is adjusted so that they will not be disturbed while making measurements. Changing the position of the leads will unbalance the circuit and the results will be misleading. The method of balancing has been mentioned under Section III, and will be further discussed in Section V.

Figure 8 is a sketch of another arrangement used for circuit 3. The brass cylinder $B$ was threaded and screwed to the binding post protruding through the bakelite bushing $B H$. This bushing passed through the case of the oscillograph. $A$ is another brass cylinder larger than $B$, and the capacitance between $A$ and $B$ was changed by sliding the brass rod $C$ through the bakelite bushing $E$. $F$ was a grounded brass cylinder surrounding the capacitor. Leakage to ground through $B H$ is objectionable for reasons previously given.

A better arrangement is to place the brass cylinder $B$, together with the other parts of the capacitor inside of the oscillograph case. The small leakage through the bakelite bushing will cause very little change in the spark discharge.

The resistance type of voltage divider was made of a number of resistances connected in series. The advantage of using a number of low resistances in series rather than a single high resistance is that a breakdown is less likely to occur and the capacitance of the unit is less, provided the same type is used for both the high and low resistance. However, this increases the capacitance to ground. This capacitance to ground may be reduced by suspending the resistors away from all metal by means of silk threads.

The spark gap used in most of the preliminary work consisted of two spheres in air. The spheres were $2 \mathrm{~cm}$ in diameter with a screw 


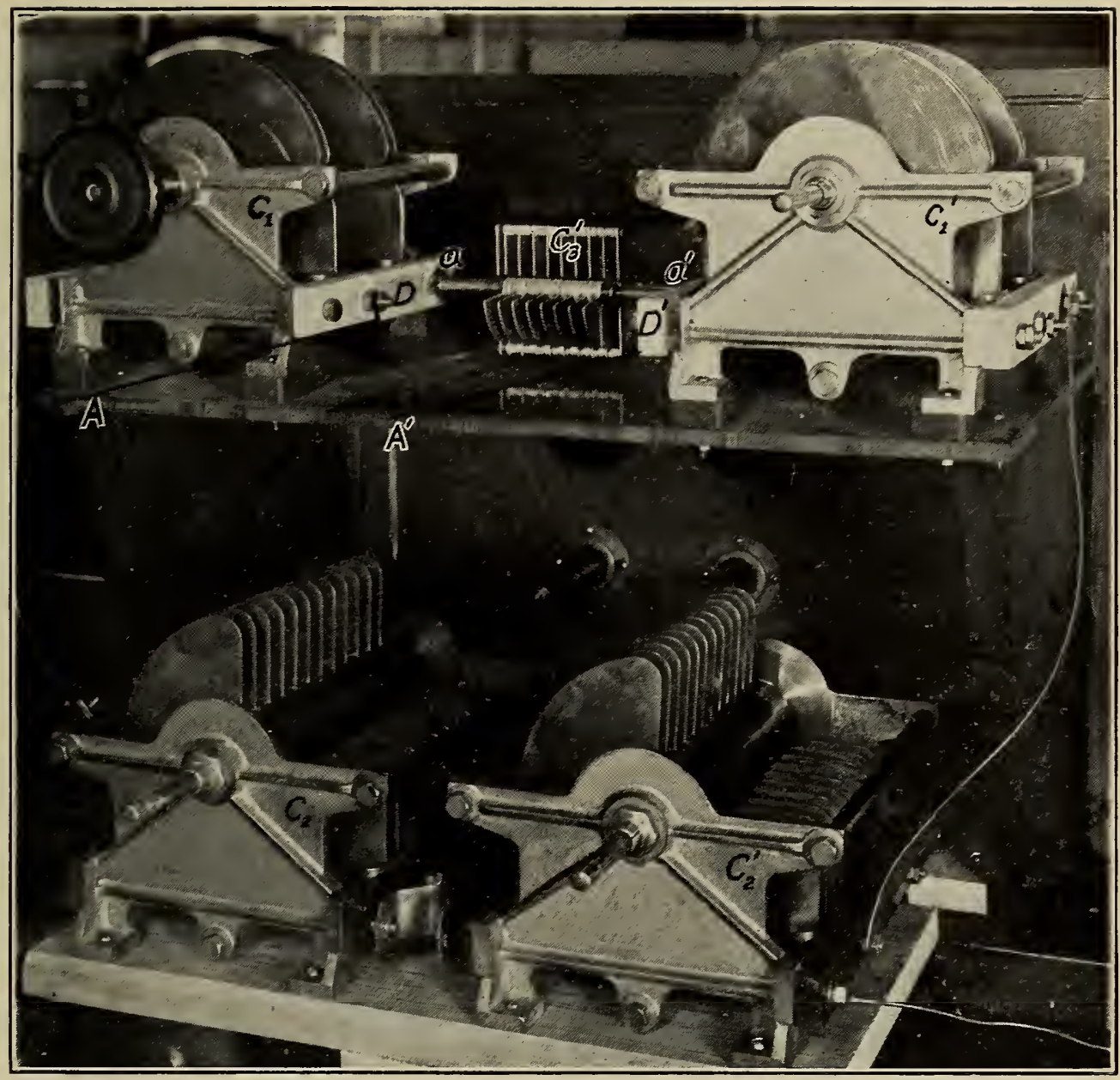

FiguRE 7.-Arrangement of capacitors for voltage divider

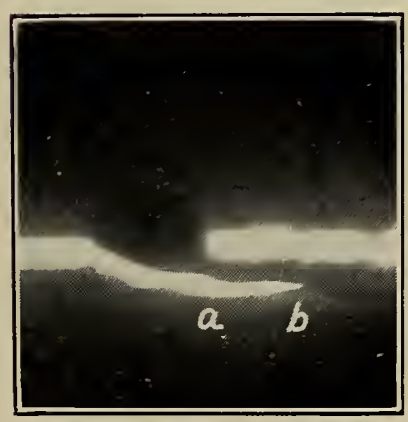

Frgure 9

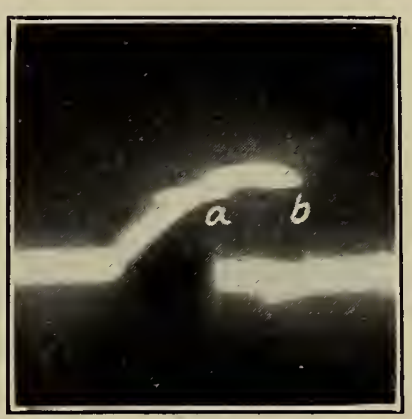

Figure 10

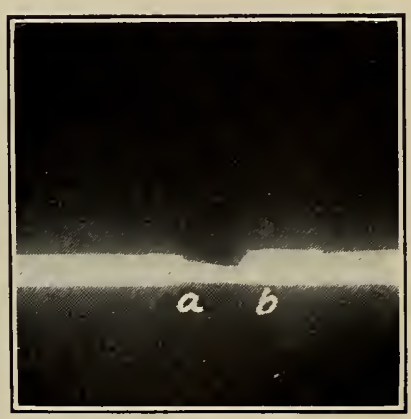

Figure 11

Oscillograms showing effect of unbalanced voltage divider. 
B. S. Journal of Research, RP460

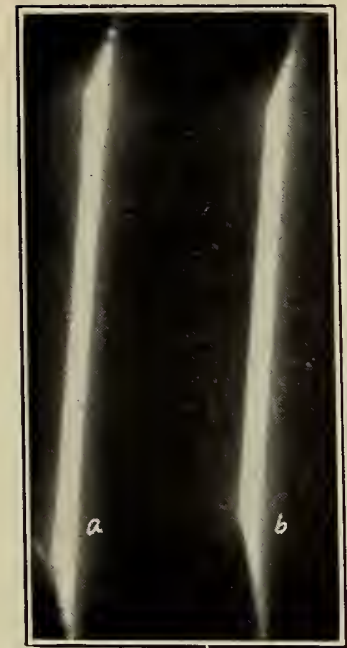

Figure 13.-Slope of traces shows the reduction ratio of circuit 4 is the same at 20 and $1,000 k c$

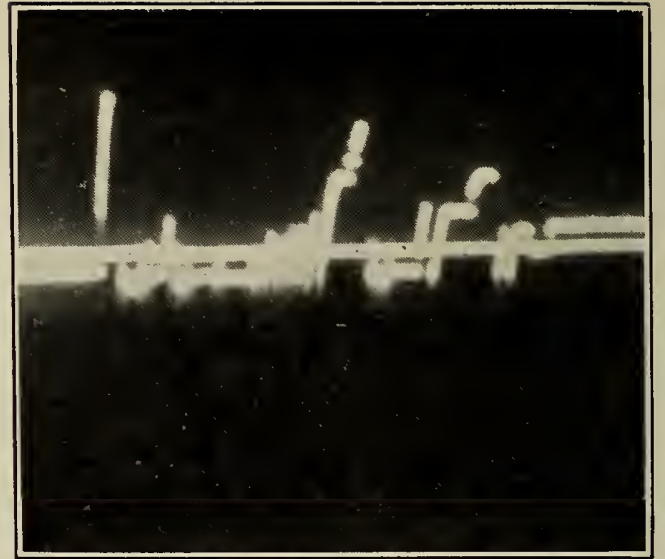

Frgure 14.-Magneto spark discharge in $\mathrm{CO}_{2}$ at 75 pounds pressure (circuit 1)

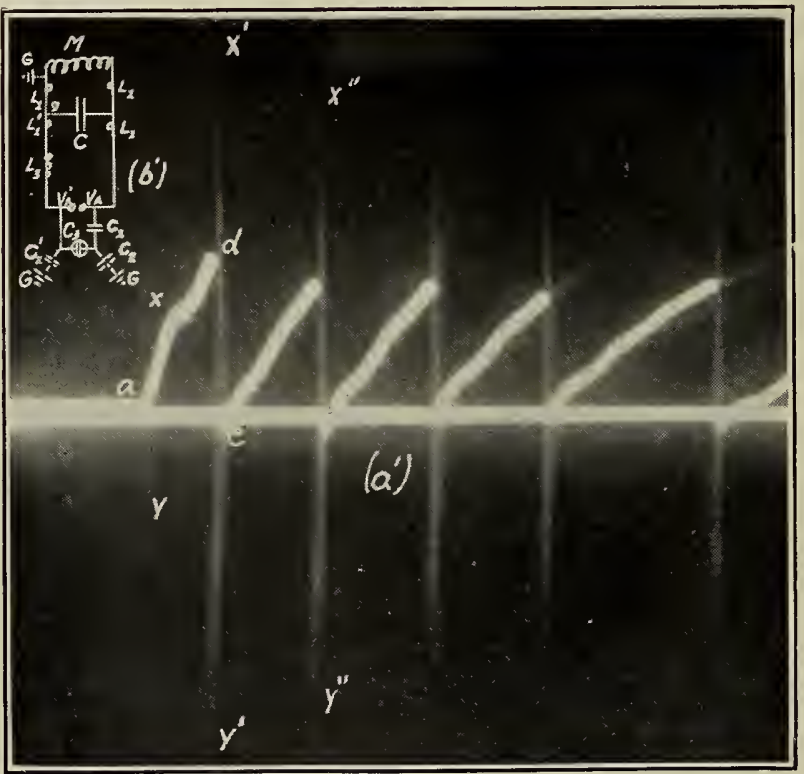

Figure 15.- $a^{\prime}$, Magneto spark discharges in $\mathrm{CO}_{2}$ at 15 pounds pressure; $b^{\prime}$, circuit arrangement used in obtaining oscillogram 
adjustment to change the gap opening. Later, measurements were made using an aviation spark plug in an atmosphere of $\mathrm{CO}_{2}$ at pressures of 15,75 , and 135 lbs./in. ${ }^{2}$ (absolute). The spark plug was screwed into the end of a brass cylinder about 4 inches long and 2 inches in diameter which was provided with a quartz window for the purpose of observing the spark.

Measurements of the high voltages used in calibrating the oscillograph were made with an electrostatic voltmeter having a range of 0 to $10 \mathrm{kv}$. A kenotron with an upper range of 30,000 volts was placed in series with the spark gap and voltmeter to ground.

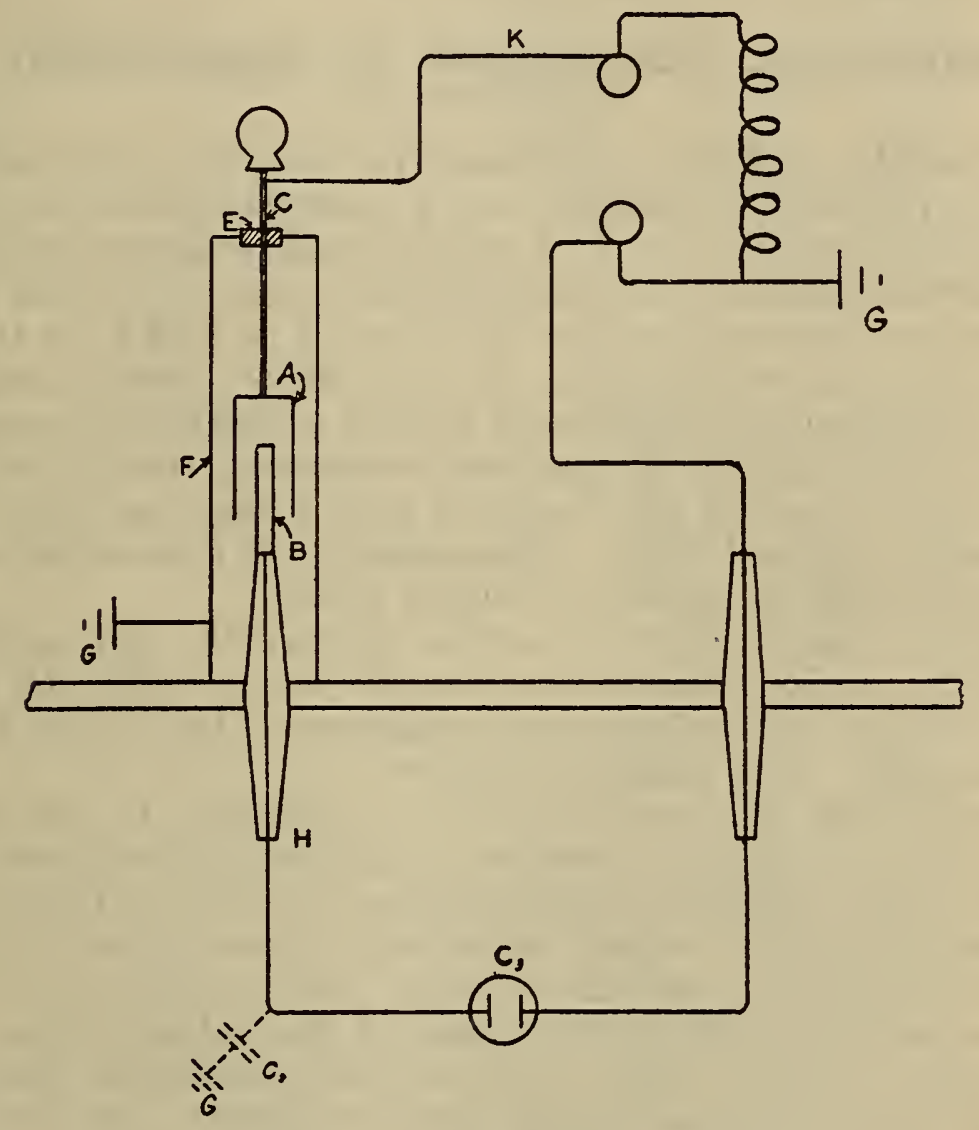

FIGURE 8.-Arrangement for circuit 2

\section{EXPERIMENTAL RESULTS WITH CIRCUITS 1 TO 5}

\section{METHOD OF BALANCING VOLTAGE DIVIDER TO SATISFY EQUATION (5)}

Before oscillograms showing the variation in voltage are taken it is necessary to balance the capacitance voltage divider as previously explained, so that the condition $\frac{C_{2}}{C_{1}}=\frac{C_{2}^{\prime}}{C_{1}^{\prime}}$ is satisfied. Since the method of balancing is the same for circuits $1,3,4$, and 5 only one set of oscillograms showing the circuit unbalanced and balanced will be given.

With the two lead wires from the divider (fig. 1) connected to the insulated side of the sphere gap, and the horizontal deflection plates in use, the oscillogram shown in Figure 9 was obtained for circuit 4. 
For correct adjustment of the position of the tube there would be no overlap of the beam as shown at $(a, b)$. This adjustment will be discussed later.

Figure 10 is an oscillogram showing the apparent difference in potential between the two lead wires when $C_{1}$ is considerably greater

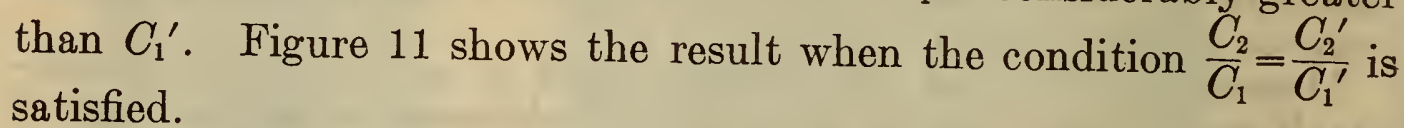

It will be understood that subsequent oscillograms pertaining to these four circuits were taken after the circuits had been balanced. It is not necessary to balance circuit 2 .

\section{EXPERIMENTAL VERIFICATION OF REQUIREMENT 2 AND}

Requirement 2, which is expressed by equation (6), states that if the voltage divider is balanced and a potential difference is applied to the leads $A$ and $A^{\prime}$ (fig. 1) a plot of peak voltage versus cathode beam deflection should give a straight line passing through the origin. This is shown to be the case for circuits $1,3,4$, and 5 by the straight lines shown in Figure 12 , at $(a),(b),(c)$, and $(d)$, respectively. These lines were obtained by applying a known potential difference to the terminals of the voltage divider and measuring the deflection. The voltage was supplied by the magneto over a range of 2,000 to 10,000 at a frequency of about $20 \mathrm{kc}$. The details as to the manner of making the calibration will be given in Section VIII.

Equipment was not available to extend the measurements of Figure 12 to higher frequencies, but it was possible to determine that the ratio of the voltages was constant in this range by the use of the oscillator. The procedure follows:

The leads from the oscillator were connected to the horizontal plates, to give a vertical deflection. Another pair of leads was connected from the oscillator to the voltage divider, the divided voltage being applied to the vertical deflection plates so as to produce a horizontal deflection. If the ratio of the applied voltage to the reduced voltage is constant, the trace of the cathode beam must be a straight line. If the ratio is to be independent of frequency the slope of the line must not change with frequency. In Figure 13 are shown two traces of the cathode beam. The straight line $(a)$ was photographed at $20 \mathrm{kc}$. and $(b)$ was photographed at $1,000 \mathrm{kc}$. As closely as measurements can be made these lines are parallel. These traces were photographed with circuit 4 , but similar results were obtained for circuits 1,3 , and 5 . Circuit 2 was not tested.

In each of these four circuits, $C_{1}$ and $C_{1}^{\prime}$ were selected so that the voltage divider did not materially change the characteristics of the circuit (requirement 1). Figures 12 and 13 show that the deflections of the cathode beam are proportional to the applied voltage and that this proportionally does not change with frequency (requirement 2). By proper selection of $C_{2}$ and $C_{2}^{\prime}$ the potentials of the deflection plates to ground were kept within the required limits (requirement 3 ). The absence of oscillations in the circuit (requirement 4) may best be determined by examining oscillograms of the spark discharge. 
3. OSCILLOGRAMS SHOWING THE VARIATION OF VOLTAGE IN MAGNETO SPARK DISCHARGES

In Figure 12 straight lines were drawn showing the relation between $V_{A}-V_{A}^{\prime}$ and the corresponding deflection of the cathode beam. These calibrations were made before the oscillograms shown in the

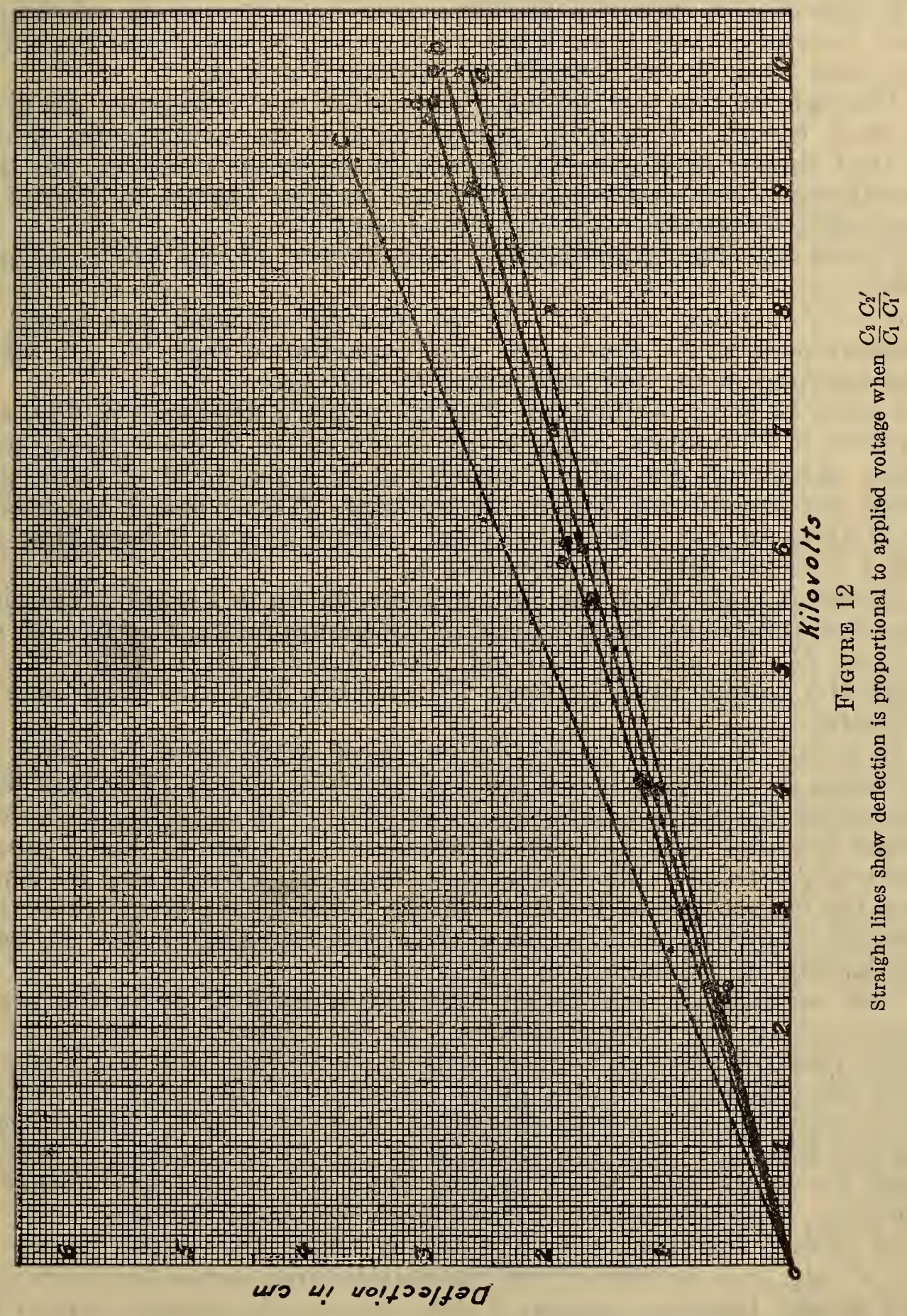

remainder of this section were taken. For this reason values of $K$ computed from Figure 12 may not agree with those which follow, since in the latter they were selected so as to give a large deflection when the spark discharge took place at a pressure of 135 pounds. With this setting of the capacitors, oscillograms were taken with 
circuits $1,3,4$, and 5 , to show the variation of voltage across a spark plug in $\mathrm{CO}_{2}$ gas during discharge. Pressures of 15,75 , and 135 pounds were used. All pressures are expressed in pounds per square inch absolute. The oscillograms for circuit 2 were taken with the spark plug in $\mathrm{CO}_{2}$ gas at 15 pounds pressure as well as with a sphere gap in air at atmospheric pressure.

In the case of circuit 4 the capacitors were purposely selected so that $V_{B}$ was greater than 1,400 volts at the higher pressures. This was done to show the necessity of keeping $V_{B}$ small.

Throughout this section only representative oscillograms are given for each circuit. It will be understood that for oscillograms similar to that shown in Figure 14 the magnetic sweep provides the time coordinate. For oscillograms similar to that shown in Figure 17 the oscillator provides the time coordinate. follow:

$C_{1}=C_{1}{ }^{\prime}=10 \mu \mu \mathrm{f}, C_{2}=C_{2}{ }^{\prime}=65 \mu \mu \mathrm{f}, C_{3}=15 \mu \mu \mathrm{f}$ (this was found by measurement to be nearer the true capacitance than $25 \mu \mu \mathrm{f}$, which was assumed in the computations), $C_{3}^{\prime}=10 \mu \mu \mathrm{f}$.

Figure 14 is an oscillogram showing a spark discharge in $\mathrm{CO}_{2}$ at a pressure of 75 pounds. This figure shows traces of the cathode beam below the sweep line which correspond to a potential of more than twice the breakdown voltage of the gap. These traces are due to oscillations in the potential divider. From this it follows that circuit 1 does not satisfy requirement 4 .

Circuit 2.- (a) With the arrangement shown in Figure 2, circuit 2, the capacitances of the six capacitors were approximately $C_{1}=10 \mu \mu$ f, $C_{1}=\infty, C_{2}=25 \mu \mu \mathrm{f}, C_{2}^{\prime}=15 \mu \mu \mathrm{f}, C_{3}=15 \mu \mu \mathrm{f}, C_{3}^{\prime}=0$. Since $C_{2}^{\prime}{ }^{\prime}$ is small the voltage drop between $V_{A}{ }^{\prime}$ and the deflection plate may be neglected.

In Figure 15, $\left(a^{\prime}\right)$ is an oscillogram taken with circuit 2 to show the variation in voltage across a spark plug in $\mathrm{CO}_{2}$ gas at 15 pounds pressure using the arrangement shown at $\left(b^{\prime}\right)$. The capacitor $C$ shunted across the spark plug and the inductances $L_{1}, L_{3}$, and $L_{1}{ }^{\prime}$ had a capacitance of $2,800 \mu \mu f$. The inductances designated by $L_{1}$ and $L_{1}^{\prime}$ represent the inductance of the leads in the parts of the circuit indicated. The inductance of each $L_{1}$ and $L_{1}^{\prime}$ was approximately $1 \mu \mathrm{h}$. $L_{3}$ was a coil having an inductance of $3 \mu \mathrm{h}$.

This oscillogram was taken with the horizontal deflection plates connected to the voltage divider. At $(a)$ the voltage began to increase and continued to increase until it reached $(d)$, where a discharge took place across the spark plug. During the discharge the voltage drop across the spark plug should be small because the resistance of the gap is small. The fainter traces at $\left(x^{\prime}, y^{\prime}\right)$ etc., indicate a voltage drop across the plug much greater than the breakdown voltage of the gap. An explanation of these large deflections follows.

If during the discharge $V_{A}-V_{A}^{\prime}$ is small the traces shown by $\left(x^{\prime}, y^{\prime}\right)$ etc., can not come from the voltage drop across the spark gap, and should, therefore, appear if both leads of the voltage divider are connected to the same side of the spark gap. Figure $16\left(b^{\prime}\right)$ shows diagrammatically the circuit arrangement used in obtaining the oscillogram shown in Figure $16\left(a^{\prime}\right)$. The traces $\left(x^{\prime}, y^{\prime}\right)$ etc., are present, and while measurements show that they are slightly larger in Figure 15 $\left(a^{\prime}\right)$ than in Figure $16\left(a^{\prime}\right)$ this is to be expected because in the first. 
case $V_{A}$ differs by a small amount from $V_{A}^{\prime}$ and in the second $V_{A}=V_{A}^{\prime}$. That this is so becomes apparent from equation (15),

$$
V_{B}-V_{B}^{\prime}=\frac{V_{A} C_{1}-V_{A}^{\prime}\left(C_{1}+C_{2}\right)}{C_{1}+C_{2}+C_{3}+C_{3}^{\prime}}
$$

for if $V_{A}=V_{A}^{\prime}$ the voltage $V_{B}-V_{B}{ }^{\prime}$ across the deflection plates will be greater or less than

$$
\frac{V_{1}^{\prime} C_{2}}{C_{1}+C_{2}+C_{3}+C_{3}^{\prime}}
$$

which is the value resulting from equation (15) when $V_{\Delta}=V_{A}^{\prime}$.

An inspection of the circuit diagram shows that during the discharge of the capacitor $C$, the drop in $\left(L_{1}^{\prime}+L_{3}\right)$ will make $V_{A}^{\prime}$ different from ground potential. $V_{\mathbf{A}}^{\prime}$ has been measured and found to be 2,300 volts. Substituting this value of $V_{A}^{\prime}$ in equation (15) for the circuit arrangement shown in Figure $16\left(b^{\prime}\right)$

$$
V_{B}-V_{B}{ }^{\prime}=\frac{-V_{A}{ }^{\prime} C_{2}}{C_{1}+C_{2}+C_{3}+C_{3}^{\prime}}=\frac{-2,300 \times 25}{50}=-1,150 \text { volts. }
$$

This corresponds to a deflection of $\frac{1,150}{250}=4.6 \mathrm{~cm}$, which agrees with the measured value of $4.7 \mathrm{~cm}$ for $\left(y^{\prime}\right)$. The measured length of $\left(x^{\prime}\right)$ is $4.5 \mathrm{~cm}$.

The faint trace $(x, y)$ is attributed to conditions arising from the breakdown of the gap in the distributor. Figure $15\left(a^{\prime}\right)$ shows that when the gap breaks down the potential of the circuit increases so rapidly that the cathode beam leaves only a faint trace. This is shown by the separation at $(a)$. This sudden application of voltage causes an appreciable difference of potential between ground and $V_{A}^{\prime}$. The measured deflection of the cathode beam is $2.0 \mathrm{~cm}$ (fig. 16 $\left(a^{\prime}\right)$ ), which corresponds to a voltage across the deflection plates of $2.0 \times 250=500$. Substituting in equation (15), $500=\frac{-V_{A}^{\prime} \times 25}{500}$ or $V_{A}^{\prime}=-1,000$ volts at the instant the distributor gap breaks down.

Figure $16\left(a^{\prime}\right)$ shows that $(a, d)$ is horizontal, and indicates that $V_{A}^{\prime}$ is zero before the spark gap breaks down. Equation (12) may then be used to compute $V_{A}-V_{A}^{\prime}$ from Figure $15\left(b^{\prime}\right)$.

Figure 17 is an oscillogram showing the traces $\left(x^{\prime}, y^{\prime}\right)$ etc., spread out to show the nature of the oscillations. The oscillator operating at $20 \mathrm{kc}$ was used to provide a time coordinate. The oscillogram was taken by connecting the deflection plates directly across a small portion of the lead $L_{1}^{\prime}$. The average frequency obtained from measurements made on this and similar oscillograms was $1.1 \times 10^{3} \mathrm{kc}$. Neglecting the effect of the magneto the computed frequency of the circuit

$$
\begin{aligned}
\left(C, V_{A}, V_{A}{ }^{\prime}, L_{3}, L_{1}\right) \text { is } \eta & =\frac{1}{2 \pi \sqrt{C\left(L_{1}+L_{1}{ }^{\prime}+L_{3}\right)}}=\frac{10^{8}}{6.28 \sqrt{28 \times 5}} \\
& =1.25 \times 10^{3} \mathrm{kc}
\end{aligned}
$$

which agrees with the measured value since the inductors $L_{1}, L_{1}{ }^{\prime}$, 
and $L_{3}$ were only approximately determined. The maximum current in the circuit is

$$
I=E \sqrt{\frac{C}{\left(L_{1}+L_{1}{ }^{\prime}+L_{3}\right)}}=3,000 \sqrt{\frac{2,800 \times 10^{-12}}{5 \times 10^{-6}}}=71 \text { amperes }
$$

where $E$ is taken as 3,000 volts, since it is the peak potential difference across the spark plug.

The drop in voltage between $(g)$ and $A_{1}{ }^{\prime}$ using the computed frequency (since the computed current was also used) is

$$
V_{A}^{\prime}-V_{\diamond}=\omega L I=2 \pi \times 1.25 \times 10^{6} \times 4 \times 10^{-6} \times 71=2,230 \text { volts }
$$

which agrees with the measured value of 2,300 volts if $(g)$ is assumed to be at ground potential.

It may be well to mention at this time that the portion of the circuit shown grounded, is the magneto. During the discharge of the capacitor the magneto base may not remain at ground potential although grounded to a water pipe with a copper strip. This departure from ground potential becomes more pronounced as the capacitance and inductance of the circuit are decreased. With circuit constants sufficiently small, so that $\eta=10^{4} \mathrm{kc}$, the maximum potential of the magneto to ground was found to be 750 volts. No measurements were made of the potential difference between the magneto base and ground, or between $(g)$ and ground, with the circuit shown in Figures $15\left(b^{\prime}\right)$ and $16\left(b^{\prime}\right)$. At this relatively low frequency, $1.25 \times 10^{3} \mathrm{kc}$, the drop in voltage between the magneto base and ground, or $(g)$ and ground would be much less.

If better agreement were desired between the computed and measured values of $V_{A}^{\prime}$, the potential of $(g)$ to ground would have to be determined and the result added to 2,230 volts.

(b) The capacitance of the arrangement shown in Figure 8 measured from $A$ to $B$ was approximately $20 \mu \mu \mathrm{f}$. The measurement included $C_{1}$ as well as the capacitance to ground which in this type of capacitor is relatively large as compared to $C_{1}$. Figure 18 is an oscillogram taken with the same circuit arrangement as shown in Figure $15\left(b^{\prime}\right)$, except a sphere gap was used instead of a spark plug. It will be noted that the deflections $\left(x^{\prime}, y^{\prime}\right)$ are not vertical. This will be discussed later.

Circuit 3.- The approximate capacitances of the six capacitors used in circuit 3 are: $C_{1}^{\prime}=10 \mu \mu \mathrm{f}, C_{1}^{\prime}=70 \mu \mu \mathrm{f}, C_{2}=25 \mu \mu \mathrm{f}, C_{2}^{\prime}=175 \mu \mu \mathrm{f}$, $C_{3}=15 \mu \mu \mathrm{f}$, and $C_{3}{ }^{\prime}=25 \mu \mu \mathrm{f}$.

The oscillogram shown in Figure 19 represents a spark discharge in $\mathrm{CO}_{2}$ at a pressure of 135 pounds. It shows very clearly just what is taking place between the secondary of the magneto, the gap in the distributor, the lead from the magneto distributor, and the spark plug.

The sweep line $\mathrm{KL}$ was taken with the four deflection plates grounded. The horizontal deflection plates were then connected to the voltage divider and the cathode beam swept across the photographic plate until it reached $(a)$ where the contact in the primary circuit was broken.

After the contact was broken the series gap in the distributor of the magneto broke down, and this raised the potential difference across the gap to the voltage indicated at $(b)$. This increase was so 

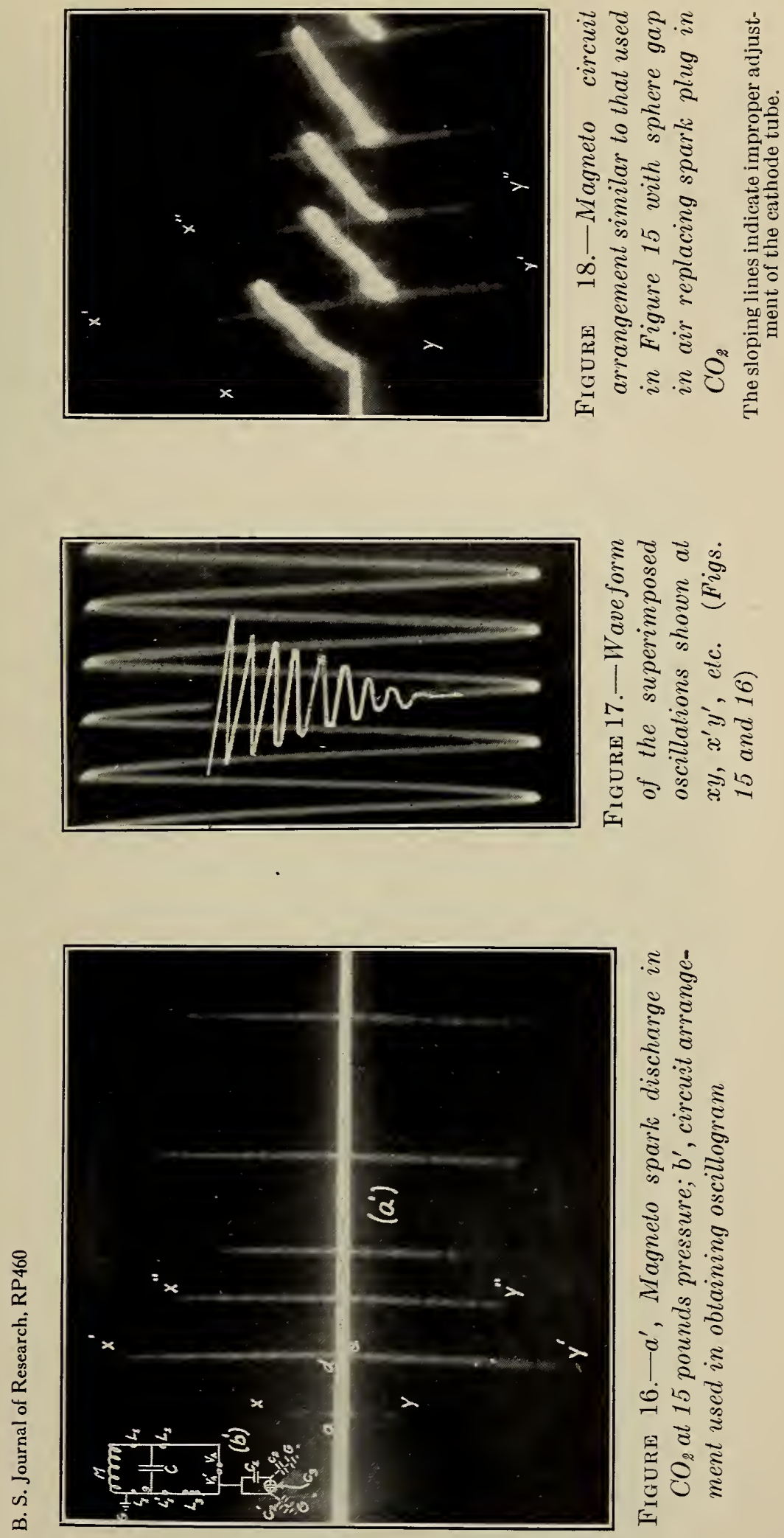


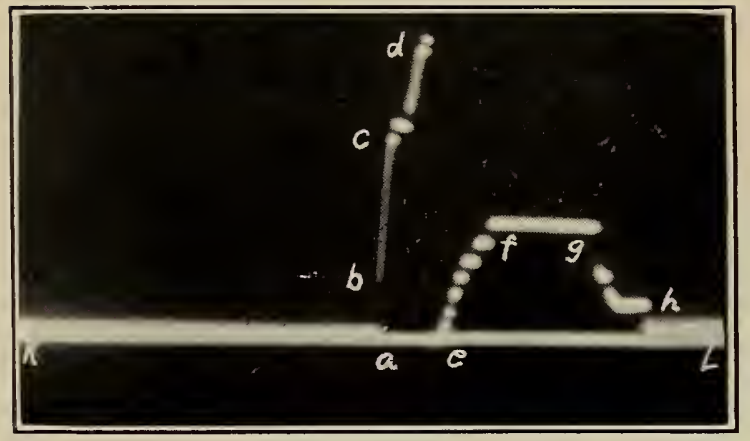

Figure 19.-Magneto spark discharge in $\mathrm{CO}_{2}$ at 135 pounds pressure (circuit 3)

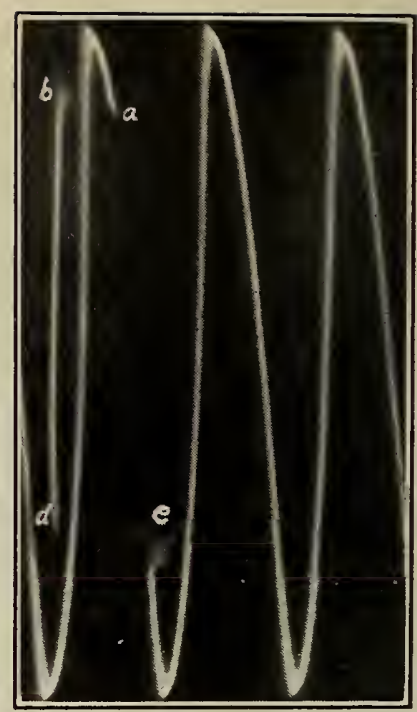

Figure 20.-Magneto spark discharge in $\mathrm{CO}_{2}$ at 15 pounds pressure (circuit 4)

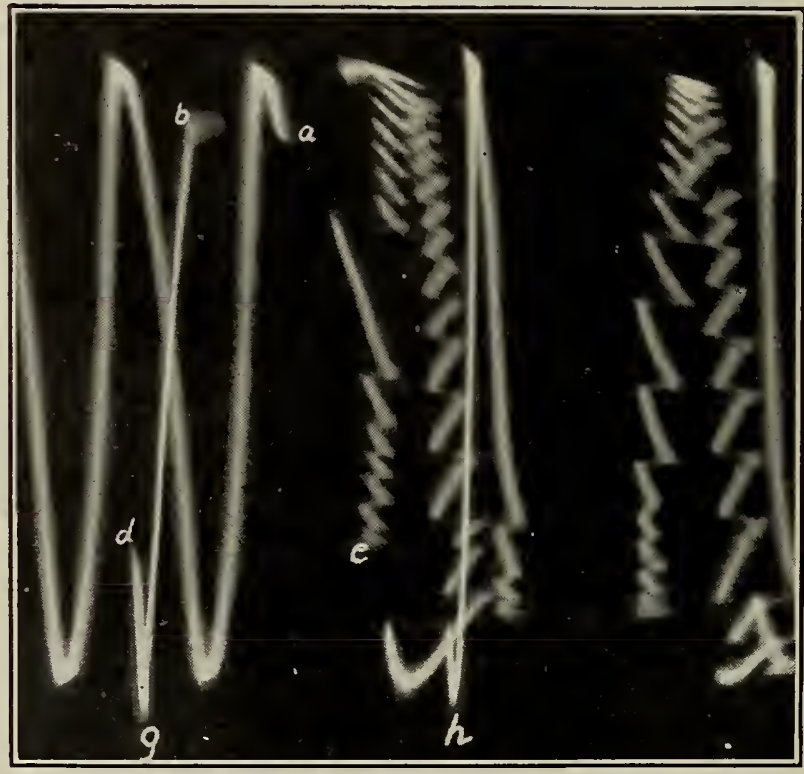

FIgURE 21.-Magneto spark discharge in $\mathrm{CO}_{2}$ at 75 pounds pressure (circuit 4)

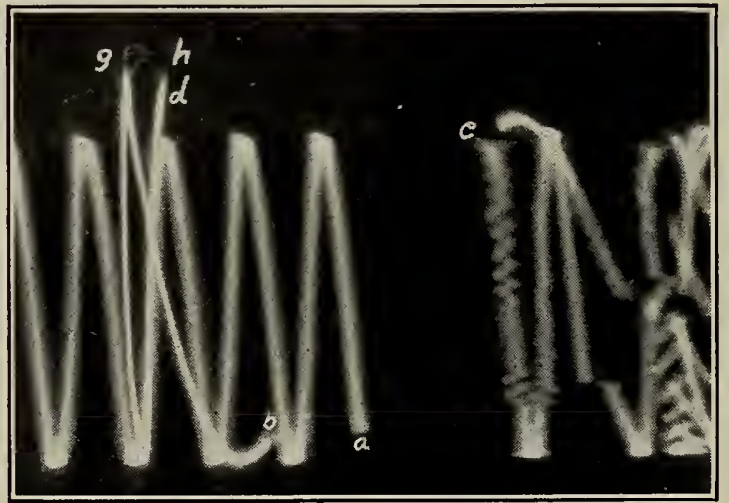

Figure 22.-Magneto spark discharge in $\mathrm{CO}_{2}$ at 135 pounds pressure (circuit 4) 


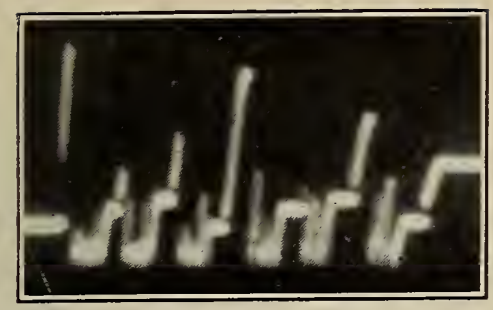

FIgURE 23.-Magneto spark discharge in $\mathrm{CO}_{2}$ at 75 pounds pressure (circuit 5)

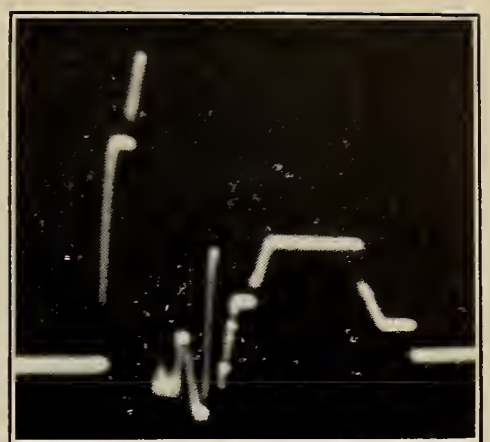

Figure 24.-Magneto spark discharge in $\mathrm{CO}_{2}$ at 135 pounds pressure (circuit 5)

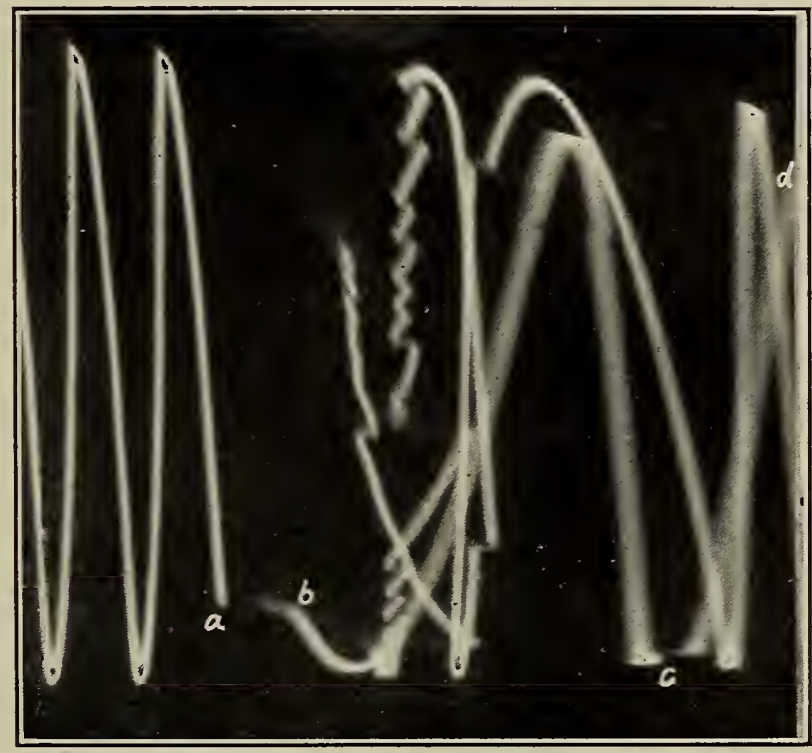

FigURE 25.-Magneto spark discharge in $\mathrm{CO}_{2}$ at 135 pounds pressure (circuit 5)

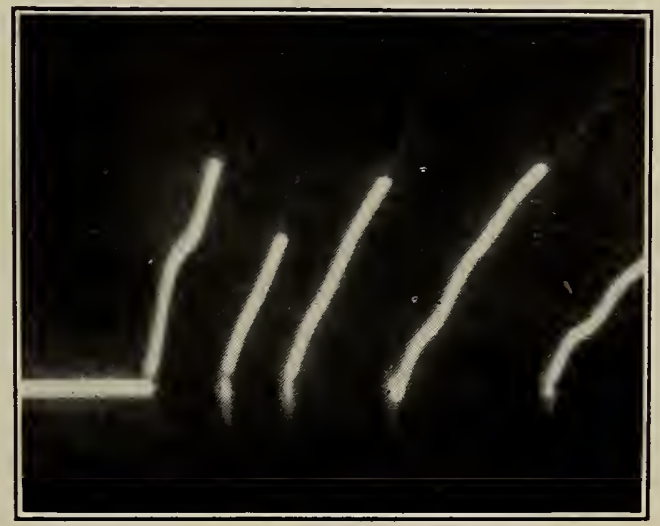

Figure 26.-Magneto spark discharge in $\mathrm{CO}_{2}$ at 15 pounds pressure (circuit 5) 
B. S. Journal of Research, RP450

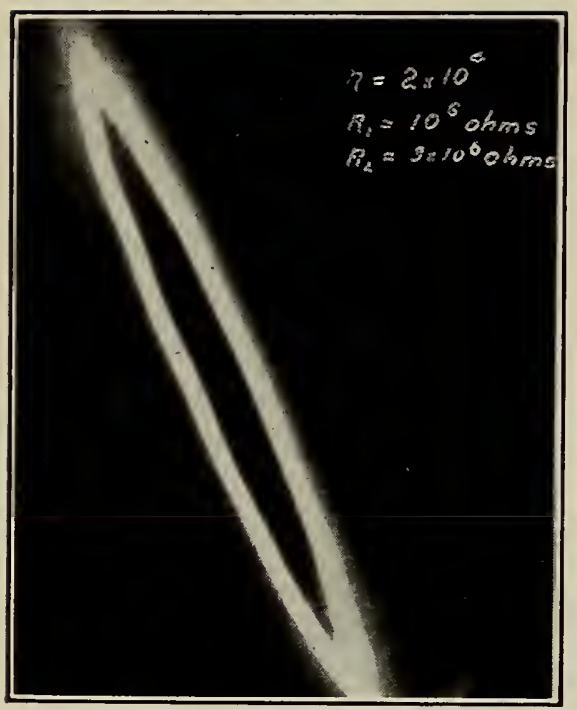

Figure 27

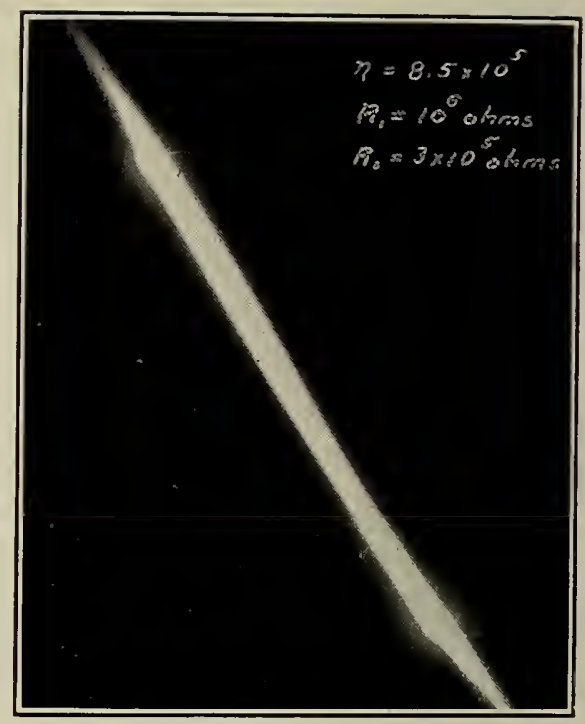

Figure 28

Change in reduction ratio and phase of applied voltage to the divided voltage using resistance voltage divider.

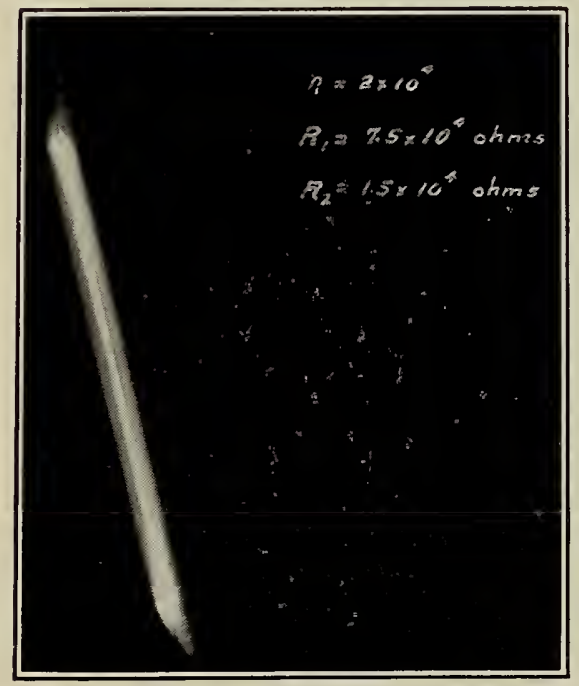

Figure 29

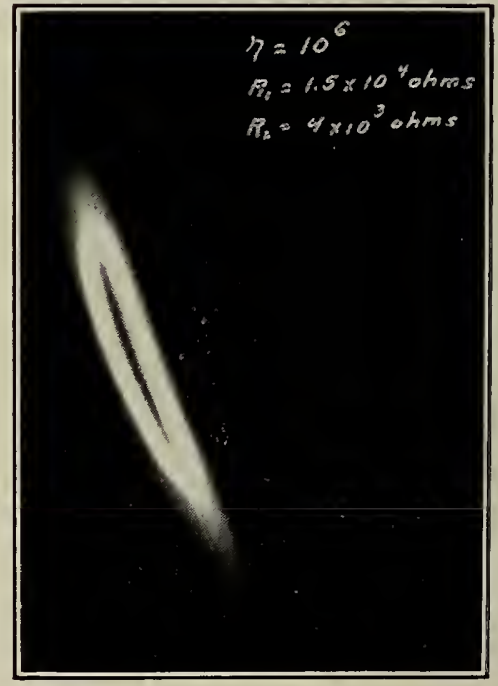

Figure 30

Change in reduction ratio and phase of applied voltage to the divided voltage using resistance voltage divider. 


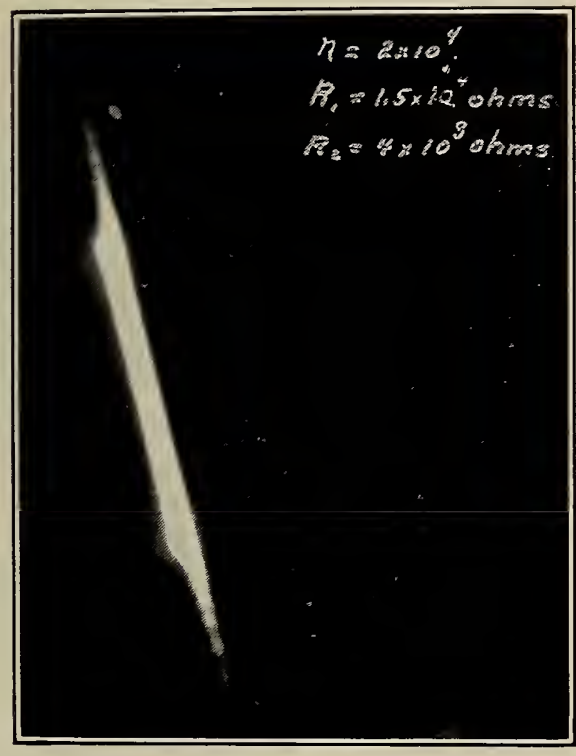

FIgURE 31

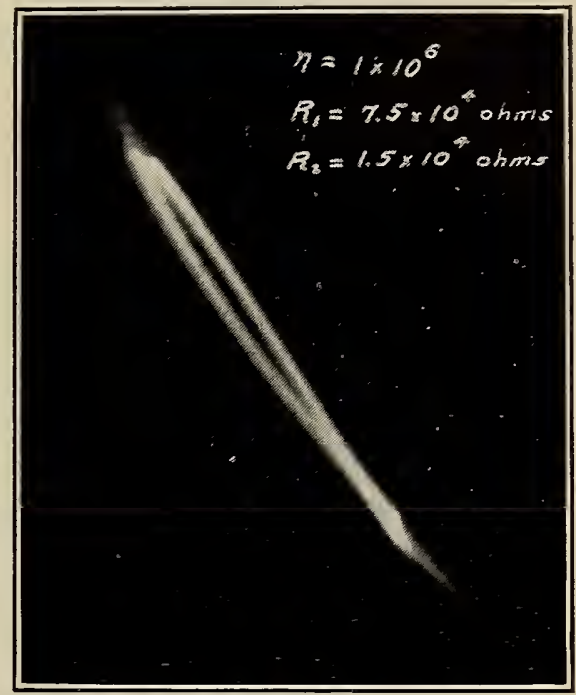

Figdre 32

Change in reduction ratio and phase of applied voltage to the divided voltage using resistance voltage divider.
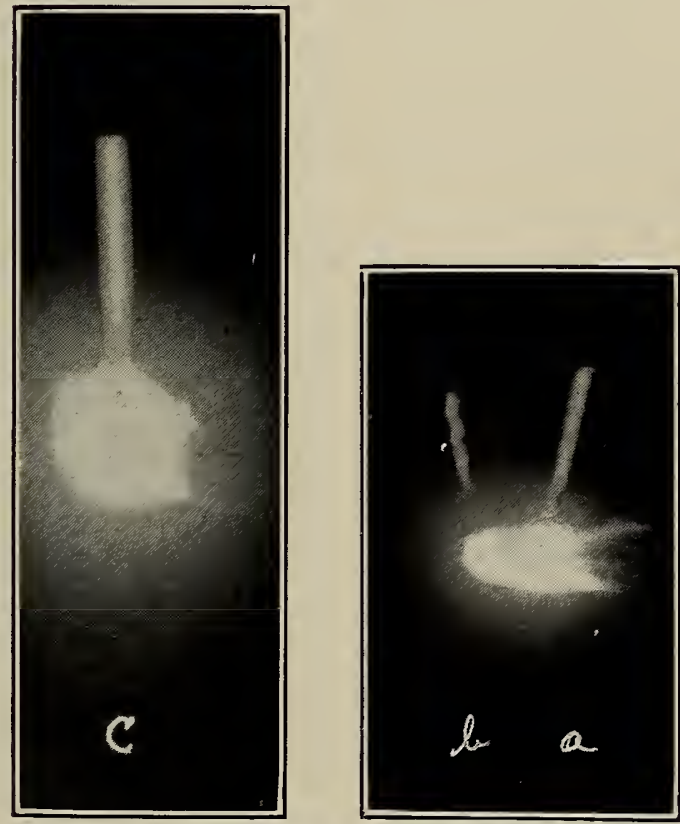

FrguRE 33

Deflections $(a)$ and $(b)$ show the effect of improper cathode tube alignment. The vertical deflection at (c) indicates a correct alignment.

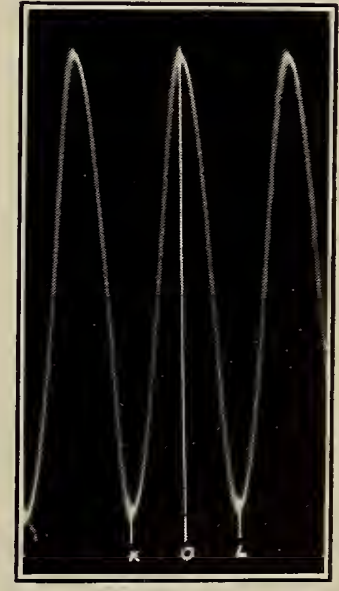

Figure 34.-Symmetrical wave form resulting from proper cathode tube alignment 
rapid that the cathode beam left no trace. The gap remained broken and the voltage increased to $(c)$ when the series gap broke down, healed, and broke down again until the voltage across the gap was indicated by $(d)$, where it healed again. The series gap again broke down and with this small increase in voltage the spark plug gap broke down. The voltage then dropped so rapidly that again the cathode beam did not leave a trace. At $(e)$ there is an indication below the sweep line of a small voltage. The spark gap in the magneto broke, and a series of discharges took place across the gap until the voltage indicated at $(f)$ was reached. The voltage in the magneto probably increased slightly, but not enough to break down the gap in the magneto. The voltage across the spark plug remained constant until finally the magneto voltage began to fall, and a discharge took place from the spark plug circuit to the magneto. This continued until $(h)$ was reached where the potential difference between the magneto and the spark plug circuit was too small to further break down the gap and the potential at $(h)$ remained on the spark plug. This voltage is also shown to exist across the spark gap when the sweep first appears on the left side of the oscillogram.

Circuit 3 shows no oscillation, so it satisfies requirement 4 . It may be used where the large capacitances $C_{1}{ }^{\prime}$ and $C_{2}^{\prime}$ are not objectionable.

Circuit 4.-The approximate capacitance of the six capacitors are $C_{1}=C_{1}{ }^{\prime}=20 \mu \mu \mathrm{f}, C_{2}=C_{2}{ }^{\prime}=25 \mu \mu \mathrm{f}, C_{3}=15 \mu \mu \mathrm{f}, C_{3}{ }^{\prime}=75 \mu \mu \mathrm{f}$.

The capacitances of $C_{1}$ and $C_{1}^{\prime}$ were increased to $20 \mu \mu \mathrm{f}$ to bring out the effect of increasing $V_{B}$ and $V_{B}{ }^{\prime}$.

Figures 20,21, and 22 are oscillograms representing spark discharges at pressures of 15,75 , and 135 pounds, respectively, in $\mathrm{CO}_{2}$ gas. For these photographs the oscillator provided a time axis. The voltage to be measured was applied to the vertical plates. The photographs are lettered in the same manner as in Figure 19. These photographs show that oscillations are set up in the voltage divider and requirement 4 is not satisfied.

In Figure 21 the peak potential of $V_{B}$ is approximtely 1,770 volts and in Figure 22 it is approximately 2,750 volts. The effect of this increased voltage is shown at $(g)$ and $(h)$. These traces indicate voltages on the deflection plates connected to the oscillator greater than the voltage of the oscillator. They are similar to those which appeared in Figures 9, 10, and 11 on the horizontal plates. These deflections are spurious, and are probably due to a potential field set up between the deflection plates $B$ and their surroundings.

Circuit 5.-The approximate capacitances of the six capacitors used in circuit 5 were: $C_{1}=C_{1}{ }^{\prime}=10 \mu \mu \mathrm{f}, C_{2}=C_{2}{ }^{\prime}=65 \mu \mu \mathrm{f}, C_{3}=15 \mu \mu \mathrm{f}$, $C_{3}{ }^{\prime}=0$. Five hundred ohm resistors were inserted at $(a)$ and $\left(a^{\prime}\right)$.

Figure 23 is an oscillogram showing the variation in voltage, the discharge taking place in $\mathrm{CO}_{2}$ gas at 75 pounds pressure. The long faint traces which appear in Figure 14 are not present in this photograph. The faint traces appearing below the heavy traces are probably due to slow electrons, since similar faint traces may be found at maximum voltage of the oscillator. (See fig. 25.)

Figures 24 and 25 are oscillograms representing spark discharges at 135 pounds pressure. Faint traces due to oscillations in the voltage divider are absent in these photographs. Figure 26 is an oscillogram taken with circuit 5 under the same sparking conditions as the os- 
cillogram shown in Figure 15 with circuit 2. The oscillations in the voltage divider which are pronounced with circuit 2 are not present with circuit 5 . The faint traces appearing at peak voltage are due to the slow electrons in the cathode beam.

Figure 25 shows a progressive shortening of the oscillator amplitude as the voltage across the vertical deflection plates increases. This will be discussed in Section VII.

\section{EXPERIMENTAL RESULTS WITH RESISTANCE VOLTAGE DIVIDERS}

It was shown theoretically in Section III that on account of the effective capacitance of resistors and their supports at high frequencies, the reduction ratio of a resistance divider, in general, changes with the frequency. Oscillograms to illustrate the change in reduction ratio as the frequency is varied are shown in Figures 27 to 32 . The oscillator voltage was applied directly to the horizontal plates and the reduced voltage to the vertical plates. In each case the total resistance was $R_{1}+R_{2}$, and the vertical deflection plates were connected across $R_{2}$.

With $R_{1}$ consisting of two 500,000 -ohm grid leaks in series and $R_{2}$ of two $150,000-\mathrm{ohm}$ grid leaks in series, the oscillogram shown in Figure 27 was taken at $20 \mathrm{kc}$ and that in Figure 28 at $850 \mathrm{kc}$. The reduction ratio measured from Figure 27 is 0.35 while from Figure 28 it is 0.47 . The low frequency reduction ratio, $\frac{R_{2}}{R_{1}+R_{2}}$, is 0.23 .

The oscillograms shown in Figures 29 and 30 were taken at 20 and $1,000 \mathrm{kc}$, respectively, with $R_{1}=15,000 \mathrm{ohms}$ (a 10,000-ohm and a $5,000-\mathrm{ohm}$ grid leak in series) and $R_{2}=4,000 \mathrm{ohms}$ (two $2,000-\mathrm{ohm}$ grid leaks in series). $\frac{R_{2}}{R_{1}+R_{2}}=0.21$. The reduction ratios measured from the oscillograms are 0.20 and 0.30 , respectively.

The oscillograms shown in Figures 31 and 32 were taken with wirewound resistors, with $R_{1}=75,000 \mathrm{ohms}$ (a single resistor) and $R_{2}=$ $15,000 \mathrm{ohms}$ (a 10,000-ohm resistor in series with one of $5,000 \mathrm{ohms}$ ). $\frac{R_{2}}{R_{1}+R_{2}}=0.17$. The measured reduction ratios are 0.17 at $20 \mathrm{kc}$ and 0.29 at $1,000 \mathrm{kc}$.

It is generally impossible to arrive at these reduction ratios by using equation (16), since neither $C_{1}$ nor $C_{2}$ are accurately known. Furthermore, equation (16) does not take into account capacitances to ground. It is sufficient for the purposes of this discussion to show on the theoretical side that the ratio $\frac{E_{2}}{E}$ may either increase or decrease with increase in frequency of the applied voltage and to show on the experimental side that this ratio does vary with the frequency.

\section{OSCILLOGRAPH TECHNIQUE}

In connection with the study of voltage dividers, certain details in the technique of cathode-ray oscillograph operation were found to be important. Some of these are mentioned in the literature of the cathode-ray oscillograph, but because of their importance it seems desirable to consider them more in detail at this time. 


\section{ALIGNMENT OF THE CATHODE TUBE}

The usual method of aligning the cathode tube is to place the bright spot in the center of the photographic plate. This means that the active spot on the electrode, the small opening or diaphragm through which the electrons enter the deflection tube and the center of the photographic plate are in a straight line. In the deflection tube the cathode beam must travel between two sets of deflection plates. When a voltage is applied to the plates an electrostatic field exists with respect to the surroundings. If the deflection plates and surroundings are symmetrical with respect to the cathode beam, the electrostatic field will have a kind of symmetry that will produce no deflection of the ray except that which is normal to, and caused by. the difference of potential between each pair of plates. If the field is not symmetrical, the voltage applied to each pair of deflection plates will produce a deflection made up of two components, one normal and one parallel to the plates.

If the two vertical deflection plates $B$ and one horizontal plate $A$ shown in Figure 6 are connected to ground and a voltage applied to the second horizontal plate shown at $A$, the deflection with the usual adjustment of the cathode tube will generally show a negligible horizontal component. If plates $A$ are used with a voltage divider, such as circuit 4 , the difference of potential between the plates may be small in comparison with the difference of the potential between the plates and ground. In this case, the horizontal component may be as great as, or greater than, the vertical component. This effect is shown in Figures 9 and 10, where the tip of the deflection is not vertically above or below the break in the sweep. Figure 11 shows a horizontal deflection although the indicated potential difference between the plates is zero. By swinging the cathode tube horizontally a position was found where the tip of the deflection did not extend more than $1 \mathrm{~mm}$ beyond the break in the sweep line. With circuit 4 it was impossible to eliminate this effect entirely when $V_{B}$ and $V_{B}{ }^{\prime}$ were large.

Another example of incorrect alignment of the cathode tube is shown in Figure 18. The lines $\left(x^{\prime}, y^{\prime}\right)$ etc., may be made normal to the horizontal deflection plates by swinging the cathode tube horizontally. The traces $\left(x^{\prime}, y^{\prime}\right)$ etc., in Figure $15\left(a^{\prime}\right)$ shows the tube alignment greatly improved. To determine whether or not the cathode tube is correctly aligned for a particular set of conditions the following procedure may be used.

With the four deflection plates at ground potential and the sweep demagnetized the cathode tube is adjusted until the spot appears in the center of the fluorescent screen. The horizontal plates are connected to the voltage divider and the divider balanced as previously described, after which the leads to the divider are connected across the spark gap. If now a spark discharge takes place across the gap the electrostatic field between the deflection plates and the surroundings is that which will exist during the photographing of the discharge. By swinging the cathode tube horizontally, a position will be found where the deflection is vertical. In Figure $33,(a)$ and $(b)$ show the tube out of alignment while $(c)$ shows the tube aligned horizontally. The oscillograms were taken with circuit 4. A corresponding procedure is followed for the vertical alignment. It is well to check both alignments after the adjustments are completed. 
Figure 34 was taken after alignment of the tube by this method. It will be noted that point $(O)$ falls midway between $(K)$ and $(L)$. This method of alignment may not always be the most desirable, because if the horizontal plates are not parallel to the trace produced by the magnetic sweep, or the vertical plates not perpendicular to this trace, the adjustment will displace the cathode tube so that the bright spot is considerably displaced from the center of the photographic plate. For measuring potential differences a small slope is not objectionable unless it is desired to determine a relation between the rate of increase and decrease of the voltage. In most cases it is possible to correct for the small slope. When it is desired to determine the instantaneous relation between current and voltage, using one pair of plates to measure the voltage and the other pair to measure the potential difference across some reactance, the pattern photographed is usually so complicated that it is difficult to make corrections for both pairs of plates. It is this latter problem that led to the method given above for the adjustment of the cathode tube. In Figure 34 the bright spot is above and to the left of the center of the photographic plate.

\section{SENSITIVITY OF THE CATHODE BEAM}

It is well known that the sensitivity of the cathode beam to either an electrostatic or an electromagnetic field changes with the cathode voltage. The maximum voltage which may be applied to the cathode before a discharge takes place is limited by the pressure and composition of the gases in the cathode tube. Indirectly, then, the sensitivity of the cathode beam depends upon the pressure and composition of gases. In measuring voltages it is therefore necessary to know that the electrostatic sensitivity at the time of photographing the phenomena is the same as at the time of calibration, or else to have available a ready means of determining the sensitivity.

Measurements made with a hot-wire pressure gauge can not be relied upon to determine sensitivity if the photographic film has not been thoroughly out gassed. Unless this out gassing is carried out in a separate container before the film is placed in the oscillograph a great deal of time is lost. The procedure generally followed until this portion of the work was undertaken was for the observer to reduce the pressure until the sweep line appeared dotted and then allow the pressure to increase until the line appeared solid. At this time the photographs were taken. Working in this manner with a film which had not been out gassed the sweep first appeared dotted with a gauge reading of 54 on film 1 and a few minutes later the sweep first appeared dotted with a gauge reading of 42 on film 6 . In common with other investigators the practice was adopted of photographing the sweep with a known voltage across the horizontal plates immediately after photographing the phenomena, and using this line as a measure of sensitivity.

This same idea was used to indicate visually the sensitivity before photographing. The deflection plates, oscillator, and a source of known voltage were connected to a double-pole double-throw switch so that either the oscillator or known voltage could be applied to the deflection plates. About 1 centimeter below the top of the fluorescent screen a narrow line was drawn with willemite. When a vacuum was obtained which gave a good solid sweep the known voltage was 
adjusted until the sweep passed through the willemite line. This line appeared green against a bluish background.

To control the sensitivity it is only necessary to apply this voltage to the deflection plates and adjust the vacuum until the sweep passes

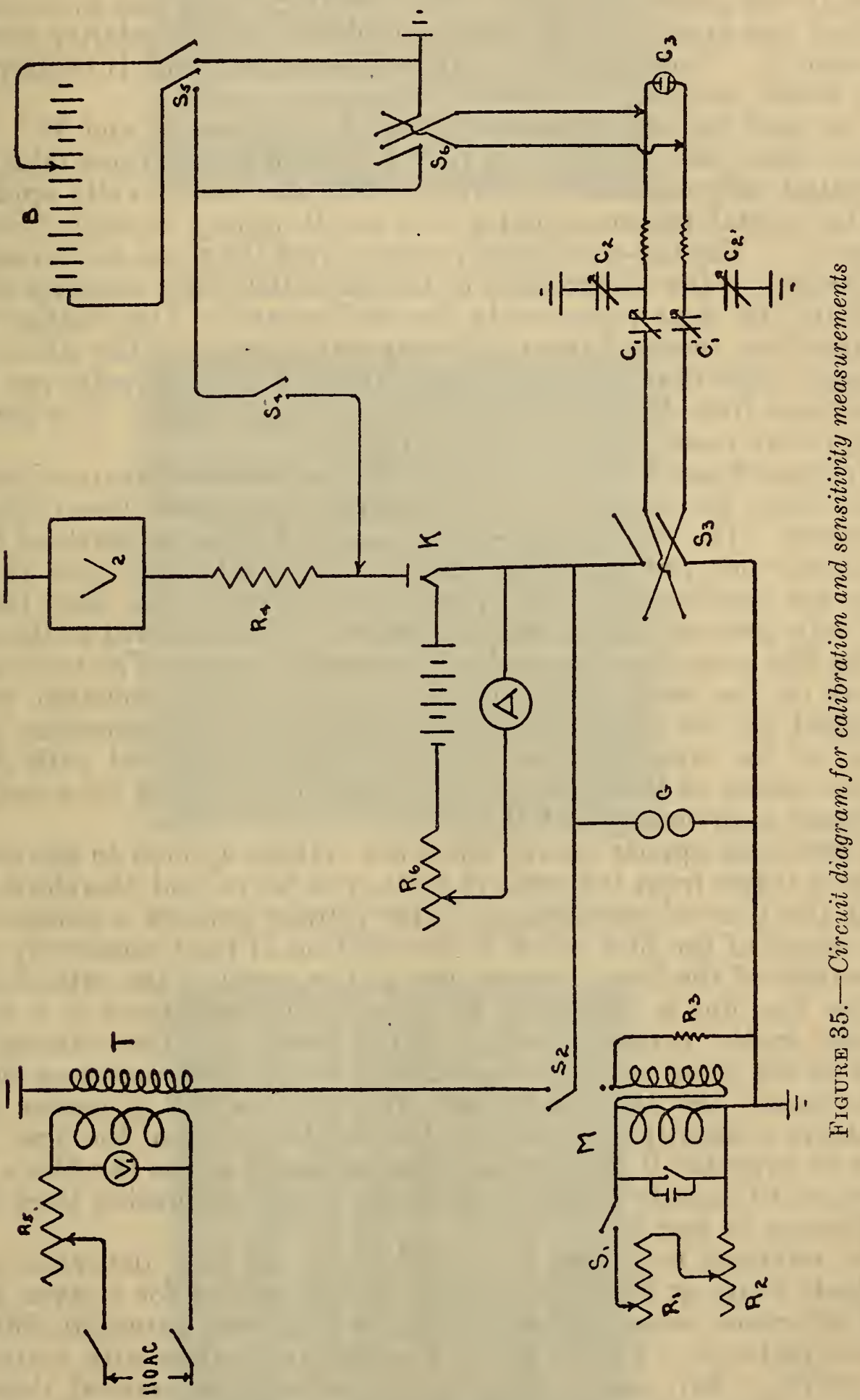

through the willemite line. The voltage was supplied by a small transformer $T$ and rectified by the kenotron $K$ as shown in Figure 35 . The double-throw switch $S_{2}$ was arranged so that the transformer or magneto could be connected to the kenotron. $V_{1}$ is an aiternating current voltmeter used to indicate the voltage drop across the primary 
of the transformer. By changing the resistance $R_{5}$ to compensate for changes in the 110 -volt supply a constant voltage was supplied to the primary. It was assumed this maintained a constant secondary voltage. The voltmeter $V_{2}$ was not sensitive enough for this work, since it was graduated in kilovolts. With $S_{5}$ open and $S_{4}$ closed, the voltage was applied to the deflection plates, and its polarity reversed, through $S_{6}$. Unless the line voltage is constant radio B batteries will give better results.

The need for such control is shown by Figures 36 and 37. These figures show only the left and right halves of oscillograms taken with potential differences of $0, \pm 218, \pm 437$, and \pm 692 volts applied to the horizontal deflection plates from the B battery through $S_{5}$ and $S_{6}$. (Fig. 35.) The hot-wire gauge readings were the same, but on account of changes in the composition of the gases the gauge readings did not indicate the same pressures in the oscillograph. This change in gas composition resulted from different out-gassing of the films. The average deflection computed from Figure 36 is 275 volts per centimeter and from Figure 37 is 215 volts per centimeter. The measurements were made at the center of the film.

Figures 38 and 39 show the left and right halves of two oscillograms taken after the sensitivity indicated by the cathode beam appeared the same. The average deflection computed from the center of Figure 38 is 250 volts per centimeter and from the center of Figure 39 is 255 volts per centimeter. The average rounded value has been taken as 250 volts per centimeter when the deflection is measured at the center of the film using the slowest electromagnetic sweep. The two extreme traces on the oscillogram are photographs of the constant voltage supplied by the transformer and kenotron to the deflection plates. None of the constant potential lines are symmetrical with respect to the center of the photograph because the cathode tube had been adjusted as given under VII, 1 .

These lines appear curved since the voltage applied to the cathode tube is taken from the peak of a 60-cycle wave and therefore varies with the time of application. The voltage reaches a maximum at the center of the film which is the position of least sensitivity. The curvature of the lines becomes less as the speed of the cathode beam across the film is increased, because in this case there is a smaller change in the cathode voltage in the interval. Observations show that as the speed at which the cathode beam is swept across the film is increased the same potential difference on the deflection plates produces a slightly greater deflection at the center of the film. This is to be expected if the cathode beam is swept across the film at such times as to include different segments of the calibration lines shown in Figures 38 and 39.

By referring to Figure 6 it will be seen that the deflection of the cathode beam at the photographic film is greater for a given potential difference across plates $B$ than for the same potential difference across plates $A$. Figure 40 is an oscillogram taken with voltages of $0, \pm 218, \pm 437$, and \pm 692 applied across the vertical deflection plates. The vertical deflection was obtained by applying the oscillator voltage to the horizontal deflection plates. The alignment of the cathode tube was the same as for Figures 38 and 39 . Measurements made between the sharp edges starting from the far central ruled lines gave an average deflection of 195 volts per centimeter. 


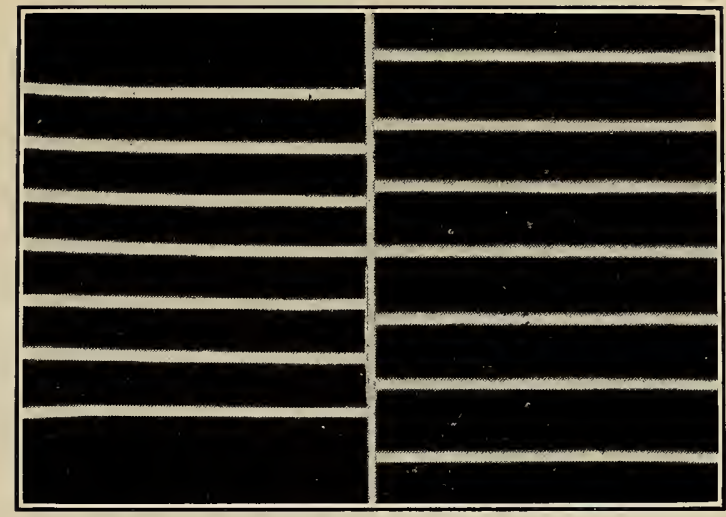

Figure 36

FIgURE 37

Two sets of calibration lines with sensitivity matched with thermocouple pressure gauge.

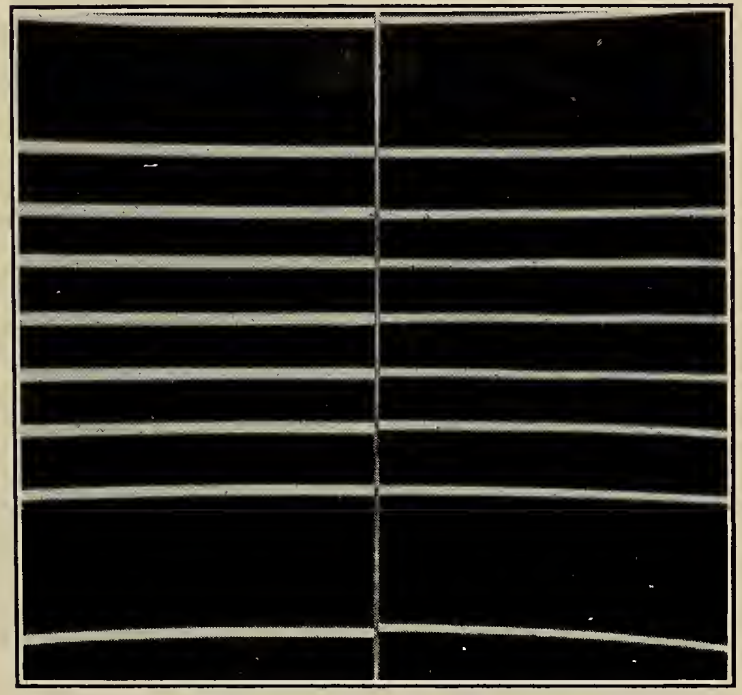

Figure 38

Figure 39

Two sets of calibration lines with sensitivity matched with known voltage and willemite mark.

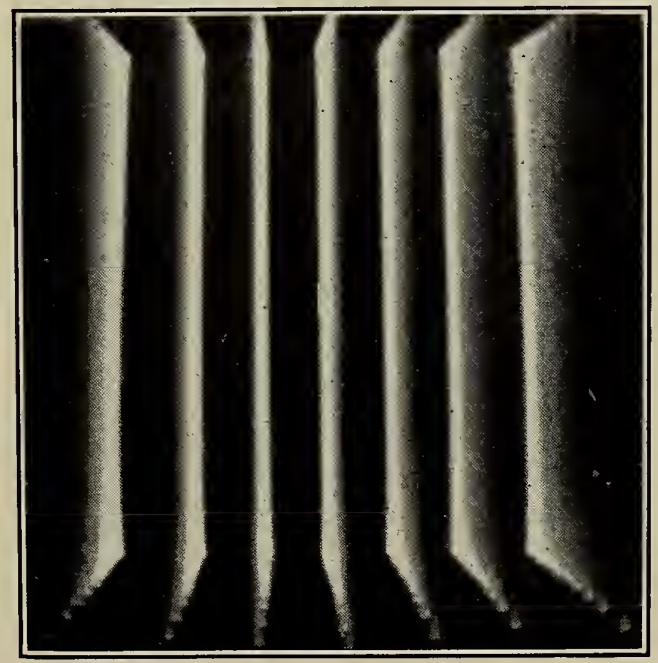

Figdre 40.-Calibration lines using vertical plates 


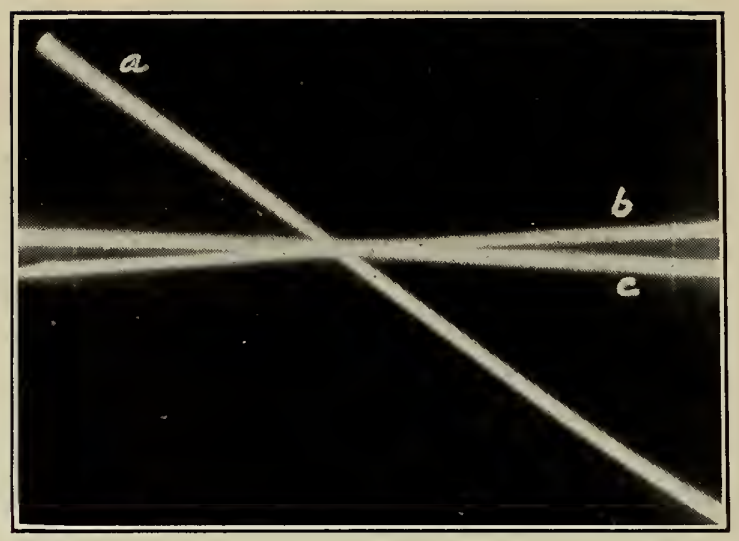

FIGURE 41

Line $a$ indicates the ratio of the sensitivity between the horizontal and vertical deflecting plates.

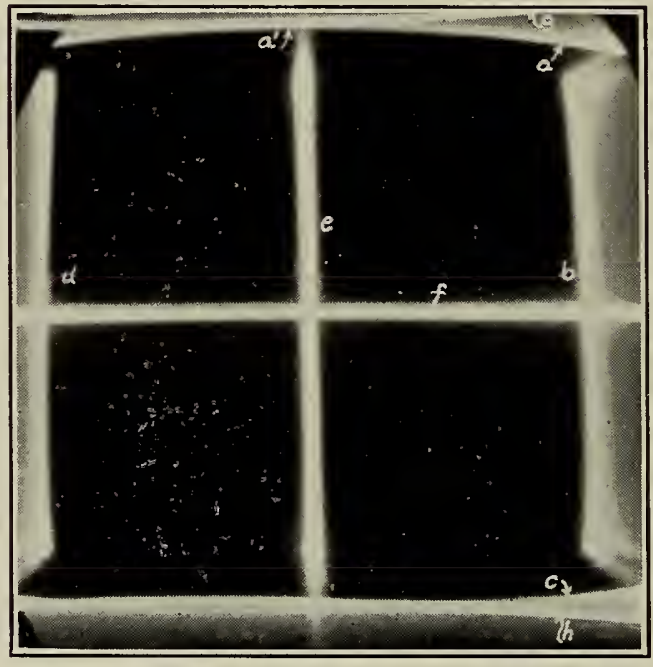

Figure 42.-Effect of nonuniform electrostatic fields

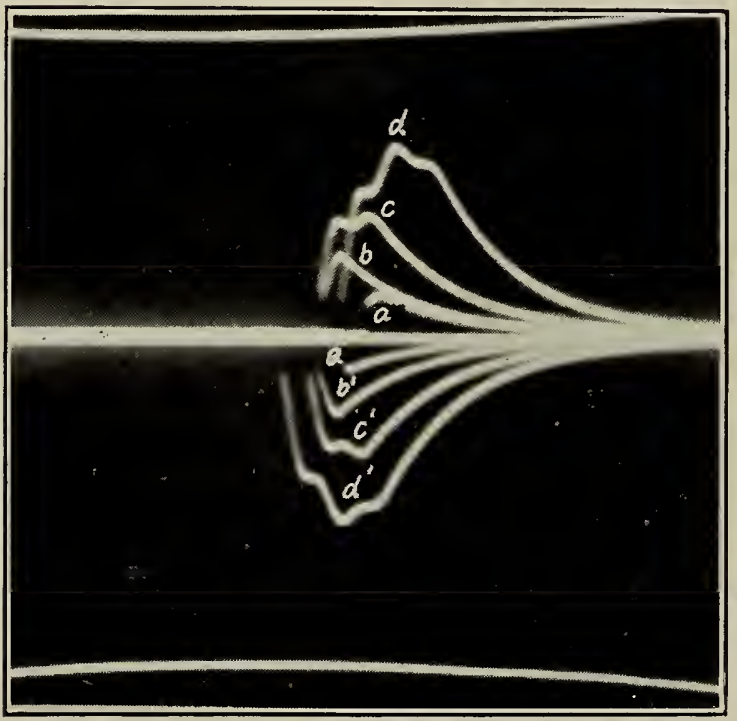

FIGURE 43.-Magneto impulses used for the calibration of voltage dividers

A 1-megohm resistor was shunted across the magneto terminals. 
B. S. Journal of Research, RP460

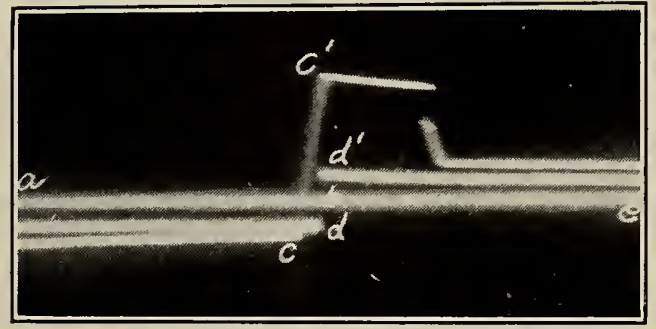

Figure 44.-Magneto impulses used for calibration of voltage divider without resistor shunted across magneto terminals

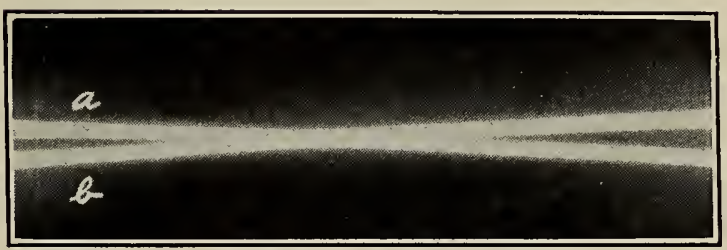

Figure 46.-Oscillogram used for determining the reduction ratio of voltage divider using oscillator 
At the time this calibration was made precautions had not been taken to control the sensitivity of the cathode beam.

A second method of determining the sensitivity of the vertical deflection plates is to determine the ratio of the sensitivity of the two pairs of deflection plates and multiply the sensitivity of the horizontal deflection plates by this ratio. If $s_{1}$ be the sensitivity of the cathode beam to plates $A$ (volts $/ \mathrm{cm}$ ) and $s_{2}$ that to plates $B$, then the ratio $v=\frac{s_{1}}{s_{2}}$ must be constant. This ratio is independent of the voltage applied to the cathode tube as well as the composition and pressure of the gases in the oscillograph. This ratio is conveniently found by applying the oscillator voltage directly to both pairs of plates. The slope of the resulting line, shown at $(a)$ in Figure 41 , is the ratio $v$, which is thus found to have the constant value 1.39 for this deflection tube. Only the straight portion of this line near the center was used because as will be shown presently a small distortion may take place with large deflections of the cathode beam. Thus if $s_{1}$ is known, $s_{2}$ is found from $s_{2}=\frac{s_{1}}{v}$. For $s_{1}=250$ volts per centimeter, $s_{2}=180$ volts per centimeter.

To explain the slight curvature at the extremities of line $(a)$ in Figure 41 as well as the progressive shortening of the oscillator amplitude in Figure 25 it is necessary to consider the space variation of electrostatic field intensity transversely across the field between the deflection plates. This variation is indicated in Figure 42. The traces $(g)$ and $(h)$ were obtained in the same manner as the two extreme calibration traces shown in Figures 38 and 39 . The trace $(a)$ was obtained by applying a constant voltage across the horizontal plates (fig. 6) and the oscillator voltage across the vertical plates. Between the vertical plates the cathode beam oscillated in a horizontal central plane at a frequency of $20 \mathrm{kc}$. The constant voltage deflected the oscillating cathode beam toward one of the horizontal deflection plates, depending upon the polarity. If the field traversed by the cathode beam is uniform the trace on the photographic film will be parallel to the plates, and if not, it will travel along a path showing the variation in field intensity. The inner edge of $(a)$ was recorded at maximum cathode voltage, which may be considered constant in this very short interval of $2.5 \times 10^{-5}$ seconds. The lighter portion above this dark line is the record of many oscillations at lower cathode voltage. The trace $(c)$ was obtained by reversing the polarity of the constant voltage. Trace $(f)$ was obtained in a similar manner after grounding the horizontal deflection plates.

The traces $(b)$ and $(d)$ were obtained by applying the constant voltage across the vertical deflection plates and the oscillating voltage across the horizontal plates. The cathode beam is first deflected to the right or left by the vertical plates, depending on the polarity of the applied voltage. The cathode beam, deflected away from the central axis horizontally, then enters the oscillating field of the horizontal plates, and oscillates at a frequency of $20 \mathrm{kc}$. With the constant voltage deflecting the beam to the right the trace $(b)$ was photographed, and with voltage reversed, $(d)$ was photographed. Trace (e) was taken in a similar manner with the vertical plates grounded. The most intense portion, of the traces, which are the inner edges of $(b)$ and $(d)$, were recorded at maximum cathode voltage. 
If it is assumed that the maximum oscillator voltage remains constant during the photographing of $(b),(e)$, and $(d)$, as well as the sensitivity as determined by the pressure and gas composition, lines joining the ends should show the change in sensitivity with transverse distance from the center of the horizontal plates. These lines would be parallel to $(a)$ and $(c)$, respectively. They would be difficult to draw because the slow electrons striking the middle of the photograhpic film fall in the same vertical plane as the fast electrons, whereas at $(b)$ and $(d)$ they are deflected to the side.

The curvature of lines $(a),(b),(c)$, and $(d)$ can be attributed to the existence of potential fields whose intensities are not uniform throughout.

It has been shown that an oscillogram taken with the oscillator voltage deflecting the cathode beam vertically and the slow magnetic sweep deflecting it horizontally, with the vertical plates grounded, would show a variation of oscillator amplitude depicted by the lines $(g)$ and $(h)$ of Figure 42 . The amount of this variation diminishes as the speed of the sweep is increased. If at the same time a voltage is applied to the vertical deflection plates, rapidly increasing to an amount sufficient to cause the horizontal deflection $(e)$ to $(b)$, the path of the beam between the horizontal plates is suddenly transferred from a field of maximum intensity $\left(a^{\prime}\right)$ to a field of less than maximum intensity $(a)$. If now the combined action of the electrostatic field of the vertical plates and the electromagnetic field of the sweep has not caused the beam to be deflected entirely off the photographic film, the resulting oscillogram will show a change in the amplitude of the oscillation. This change in amplitude will depend upon the horizontal displacement of the cathode beam by the voltage across the vertical deflection plates as well as the instantaneous cathode voltage. These two effects may either counteract or reinforce each other. Effects of this kind are believed to be the cause of the progressive shortening of the amplitude of the oscillation at $(a),(b),(c)$, and $(d)$ shown in Figure 25. In order that the shortening in Figure 25 be explained quantitatively by Figure 42 it is necessary to assume that the cathode beam in the former is displaced more to the right than the displacement shown in the latter figure.

While it is possible to explain qualitatively, at least, the lengthening of $(g)$ and $(h)$ in Figures 21 and 22 in the same manner, it is believed that these spurious effects were caused by $V_{B}$ and $V_{B}{ }^{\prime}$ becoming large. This seems all the more reasonable since a lengthening appeared in Figure 11 with no potential difference between the deflection plates. It is also now apparent why the extremities of line (a) in Figure 41 are slightly curved.

It is not intended to discuss these effects in detail or make a study of the variation of voltage throughout the whole field, but peculiar effects were obtained and their interpretation was difficult until the oscillogram shown in Figure 42 was taken.

\section{CALIBRATION OF THE CAPACITANCE VOLTAGE DIVIDER}

If the capacitance of each capacitor and lead wire were accurately known, the quantity represented by $K$, which has been called the reduction factor of the voltage divider, could be calculated. If this 
method is followed, care must be taken in measuring the capacitances of the six capacitors as well as the leads between the capacitors. The disadvantage of this method is that it requires a series of careful measurements with each change in the settings of the capacitors.

It is more feasible, therefore, to calibrate experimentally, and this may be done in two ways: (1) By applying a known alternating voltage across the divider and photographing the resulting cathode beam deflection, and (2) by determining the ratio between the cathode beam deflection when an alternating voltage is applied directly to one pair of plates and the reduced voltage to the other pair.

\section{CALIBRATION BY APPLICATION OF KNOWN VOLTAGES}

The leakage which takes place through the insulation of the capacitors prevents the use of a constant voltage for calibrating a capacitance voltage divider. A convenient source of alternating voltage is found in the magneto, since the polarities of consecutive impulses are of opposite sign. The circuit connections for calibrating are shown in Figure 35. The electrodes $G$ were separated to prevent a spark discharge across the gap. The voltage induced in the secondary of the magneto $M$ was controlled by shunting variable resistances $R_{1}$ and $R_{2}$ across the primary breaker contacts. This voltage was applied to the divider through switches $S_{2}$ and $S_{3}$, the latter switch controlling the polarity applied to the deflection plates. The kenotron $K$ acted as a check valve and allowed the voltmeter $V_{2}$ to indicate the peak voltage. The magneto, which was synchronized with the oscillograph, generated 36 surges per second, 18 positive and 18 negative with respect to ground.

Figures 43 and 44 are oscillograms showing the application of this method with and without the resistor $R_{3}$ shunted across the secondary of the magneto. Voltage divider circuit 5 was used. In Figure 44 the peak voltages indicated by the voltmeter were 2,000 and 6,000 , and are, respectively, represented by $\left(b d^{\prime}\right)$ and $\left(b c^{\prime}\right)$, where $(b)$ lies on the line of zero potential difference. The corresponding voltages of opposite polarity remaining from the previous surge are $(d b)$ and $(c b)$.

The advantage of using the 1 -megohm grid leak $R_{3}$ is shown by Figure 43. In this case the potential difference across the gap is quickly brought to zero. The surges were all of the same polarity with respect to the magneto, but their polarity with respect to the deflection plates was reversed on each voltage by switch $S_{3}$. (Fig. 35.) The peak voltages represented by $(a)$ and $\left(a^{\prime}\right),(b)$ and $\left(b^{\prime}\right),(c)$ and $\left(c^{\prime}\right)$, and $(d)$ and $\left(d^{\prime}\right)$ are, respectively, $\pm 2,350, \pm 4,075,+5,900$ and $-6,075$, and $+9,600$ and $-9,750$. These deflections determine the lines $(a)$ and $(b)$ shown in Figure 45 . Since no two surges are exactly alike and the voltmeter indicates the average of 18 surges per second, a variation between observations is to be expected. Before photographing the surges shown in Figure 43, the sensitivity was checked in the manner described in Section VII.

With a smaller reduction ratio the straight line $(d)$ in Figure 45 was determined. The plotted points represent the average of six independent observations. They were taken to show that the reduction factor of circuit 5 is constant, and that the deviations of the 
plotted points in Figure 12 become less as a greater number of observations are averaged. These deviations are caused by the magneto since the peak voltages of two sparks are not necessarily the same.

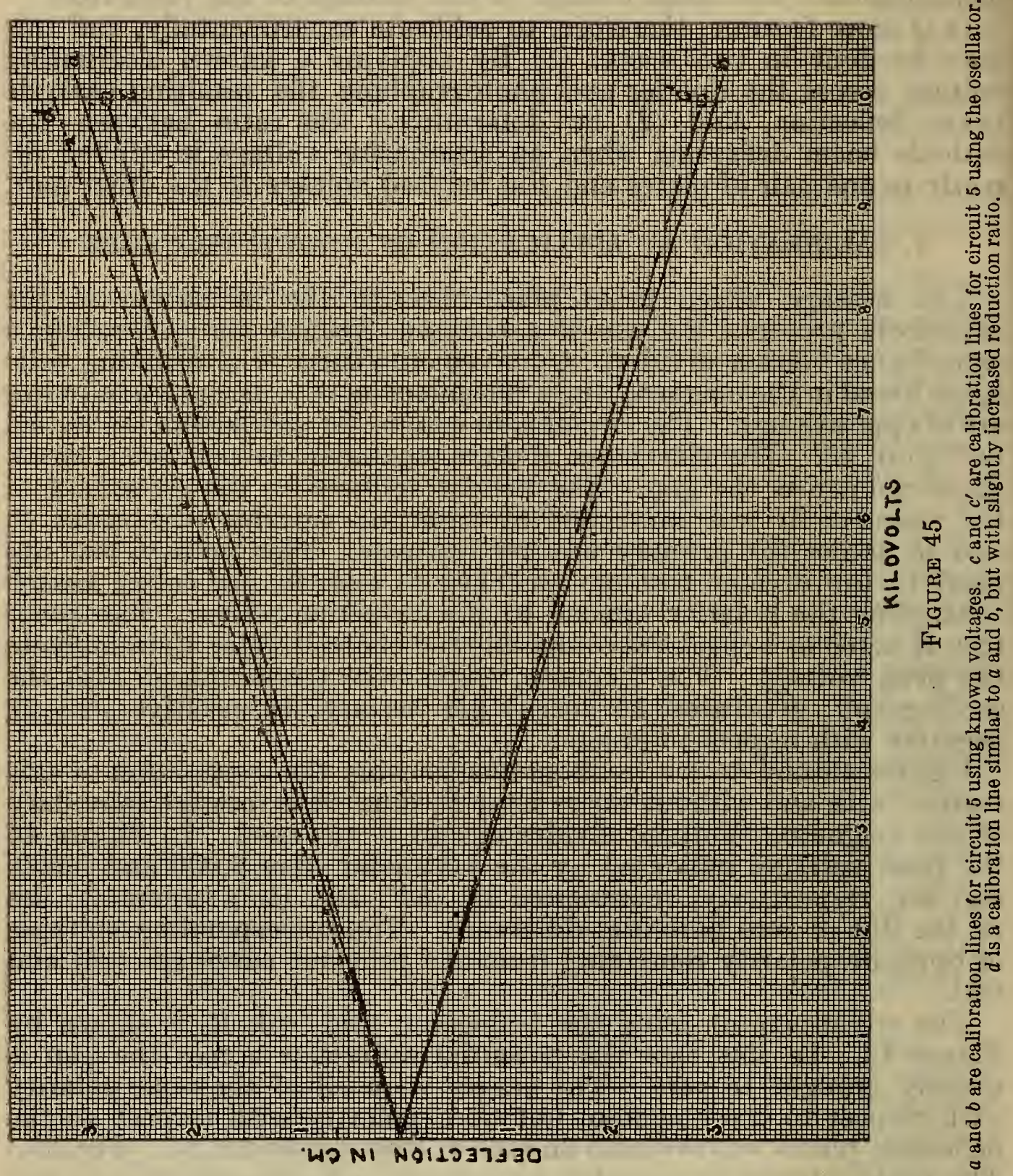

\section{CALIBRATION BY USE OF THE OSCILLATOR}

A method for using the oscillator supplied with the oscillograph to determine the relative sensitivity of the two pairs of deflection plates has already been discussed. This method, which consists simply in applying the undivided oscillator voltage to one pair of plates and the divided voltage to the other pair, is also useful for calibration. The slope of the resulting line, of which Figures 13 and 46 are examples, determines the ratio

$$
\frac{V_{B}-V_{B}^{\prime}}{V_{A}-V_{A}^{\prime}}
$$

after the ratio of sensitivity between the two pairs of plates has been determined. 
In Figure 46 the straight line $(a)$ was obtained by applying the oscillator voltage directly to the vertical deflection plates and the divided voltage (using circuit 5) to the horizontal plates. The same capacitor settings were used as for Figure 43, the reversal of slope being obtained by reversing the leads to the voltage divider. The exposures shown at $(b)$ and $(c)$ in Figure 41 were made after making a small change in the capacitor settings. With the divided voltage applied to the horizontal plates, as in this instance, the reduction factor $K$ is equal to $\frac{v \delta_{2}}{\delta_{1}}$, where $\delta_{1}$ is the mean of two deflections measured along two vertical ruled lines, $\delta_{2}$ is the total horizontal deflection measured between these lines, and $v=\frac{s_{1}}{s_{2}}=$ the ratio of sensitivity for plates $A$ (horizontal) in volts per centimeter to that for plates $B$ (vertical). The constant $v$ is 1.39. Measurements made on Figure 46 gives $\delta_{1}=0.55 \mathrm{~cm}$ and $\delta_{2}=10.75 \mathrm{~cm}$. Hence, $\frac{1}{K}=0.071$.

On account of the small slope produced, when $K$ becomes greater than 15 or 20 the method just described may not be found practicable. In such cases it will be necessary to calibrate by the method of applying known voltages. But the convenience of using the auxiliary equipment supplied with the oscillograph makes the second method preferable when the reduction ratio is of such magnitude as to allow its use. It is then unnecessary to obtain a source of high alternating voltage or high-voltage measuring instruments. (The term "high voltage" is relative. The maximum voltage used in this investigation was 10,300 .)

It is interesting to note that method (2) gives

$$
\frac{1}{K}=\frac{V_{B}-V_{B}^{\prime}}{V_{A}-V_{A}^{\prime}}
$$

absolutely, without regard to the sensitivity of the cathode beam.

To compare the results obtained by the two methods of calibration it is only necessary to substitute this value of $K$ in the expression,

$$
D=\frac{V}{K s}
$$

where $D$ is the deflection in centimeters, $V$ is an arbitrary applied voltage and $s$ is sensitivity in volts per centimeter. Let $V=10,000$ ? From the previous sensitivity measurements (Sec. VII) it is known that

and

$$
s_{1}=250 \text { volts per centimeter }
$$

$$
s_{2}=180 \text { volts per centimeter }
$$

and for the same capacitor settings $\frac{1}{K}$ was found above to be 0.0712 .

Therefore

and

$$
D_{1}=0.071 \times \frac{10,000}{250}=2.85 \mathrm{~cm} \text { for plates A }
$$

$$
D_{2}=0.071 \times \frac{10,000}{180}=3.95 \mathrm{~cm} \text { for plates B }
$$


The deflection $D_{1}$ is indicated on Figure 45 by the lines $(c)$ and $\left(c^{\prime}\right)$, and agrees closely with the calibration by the method of known potentials. If the leads had been reversed and another straight line obtained the average of the two would give a better value for $v$ than was obtained from the single line $(a)$ in Figure 41 . This should lead to a better agreement between the two methods.

\section{CONCLUSION}

The results of this investigation show that if it is desired to measure voltages in spark discharges whose peak voltages lie within the range found in the average automotive engine ignition system, voltage divider circuit 5 will probably be found satisfactory for use with a cathode ray oscillograph of the type described. However, it is well to call attention to the fact that some of the other circuits may be equally satisfactory for other types of measurements. The faint traces which were observed in oscillograms due to oscillations in the voltage divider circuit can probably be eliminated by inserting resistances as was done in circuit 5 . One of the chief merits of the capacitance voltage divider is its adaptability to a wide range of conditions of voltage measurement.

Resistance voltage dividers are unsatisfactory for frequencies commonly found in spark discharges unless special care is taken to balance the capacitance and inductance of the resistance. In the development of the equation for resistance voltage dividers only the simplest assumptions were made as to capacitance, and the inductance of the resistors was completely neglected. This equation, therefore, represents only in a very rough way what actually occurs. The oscillograms obtained in testing the resistance divider are evidence in themselves of the unsuitability of this type of divider for highfrequency work.

In the literature of cathode ray oscillography there has been a disappointing lack of detail regarding the theory and technique of measurements. It is hoped that the details set forth in this paper will be of value to those who are using the instrument in work of a similar nature.

Another point that can hardly be overemphasized is the fact that in this work no phenomena have been recorded by the oscillograph that have not been susceptible of rational explanation. It has been the repeated experience of the authors that recorded phenomena which are most baffiing when first observed on an oscillogram can usually be interpreted after careful study and experimental investigation.

\section{ACKNOWLEDGMENTS}

This work was supported by the Bureau of Aeronautics of the Navy Department and the National Advisory Committee for Aeronautics.

The authors wish to thank Doctors Mueller, Silsbee, and Brickwedde for their many helpful suggestions.

Washington, April 8, 1932. 